\title{
REVIEW
}

\section{Cerium oxide nanoparticle: a remarkably versatile rare earth nanomaterial for biological applications}

\author{
Can Xu and Xiaogang Qu
}

Cerium oxide nanoparticles (CeONPs) have received much attention because of their excellent catalytic activities, which are derived from quick and expedient mutation of the oxidation state between $\mathrm{Ce}^{4+}$ and $\mathrm{Ce}^{3+}$. The cerium atom has the ability to easily and drastically adjust its electronic configuration to best fit its immediate environment. It also exhibits oxygen vacancies, or defects, in the lattice structure; these arise through loss of oxygen and/or its electrons, alternating between $\mathrm{CeO}_{2}$ and $\mathrm{CeO}_{2-x}$ during redox reactions. Being a mature engineered nanoparticle with various industrial applications, CeONP was recently found to have multi-enzyme, including superoxide oxidase, catalase and oxidase, mimetic properties that produce various biological effects, such as being potentially antioxidant towards almost all noxious intracellular reactive oxygen species. CeONP has emerged as a fascinating and lucrative material in biological fields such as bioanalysis, biomedicine, drug delivery, and bioscaffolding. This review provides a comprehensive introduction to CeONP's catalytic mechanisms, multi-enzyme-like activities, and potential applications in biological fields.

NPG Asia Materials (2014) 6, e90; doi:10.1038/am.2013.88; published online 7 March 2014

Keywords: Alzheimer's disease; antioxidant; cerium oxide nanoparticle; enzyme; lanthanide

\section{INTRODUCTION}

Rare earth, ${ }^{1}$ which has been called an 'industrial vitamin' and a 'treasury' of new materials, has an increasingly important role in technical progress and the development of traditional industries, and it is also widely applied in high-technology industries such as information and biotechnology. ${ }^{2}$ The chemistry of rare earth differs from main group elements and transition metals because of the nature of the $4 f$ orbitals, which are 'buried' inside the atom and are shielded from the atom's environment by the $4 d$ and $5 p$ electrons. ${ }^{1,2}$ These orbitals give rare earth unique catalytic, magnetic and electronic properties. These unusual properties can be exploited to accomplish new types of applications that are not possible with transition and main group metals.

Cerium, which is the first element in the lanthanide group with $4 f$ electrons, has attracted much attention from researchers in physics, chemistry, biology and materials science. When combined with oxygen in a nanoparticle formulation, cerium oxide adopts a fluorite crystalline structure that emerges as a fascinating material. ${ }^{3}$ This cerium oxide nanoparticle (CeONP) has been used prolifically in various engineering and biological applications, such as solid-oxide fuel cells, ${ }^{4}$ high-temperature oxidation protection materials, ${ }^{5}$ catalytic materials, ${ }^{6,7}$ solar cells ${ }^{8}$ and potential pharmacological agents. ${ }^{9}$ Although useful because of its various properties and applications, the main application of CeONPs is in the field of catalysis, and stems from their unique structure and atomic properties compared with other materials. In recent years, CeONP and CeONP-containing materials have come under intense scrutiny as catalysts and as structural and electronic promoters of heterogeneous catalytic reactions. ${ }^{6}$ In industry, it has been most widely used as an active component in processes such as three-way catalysts ${ }^{7}$ for automobile exhaust-gas treatments, oxidative coupling of methane and water-gas shift reaction. Recently, CeONP has been reported to have multienzyme, including superoxide oxidase, catalase and oxidase, mimetic properties, and has emerged as a fascinating and lucrative material in biological fields such as in bioanalysis, ${ }^{10-15}$ biomedicine, ${ }^{9}$ drug delivery ${ }^{16,17}$ and bioscaffolding. ${ }^{18,19}$ This review provides a comprehensive introduction to CeONP's catalytic mechanisms, multienzyme-like activities and potential applications in biological fields.

\section{SYNTHESIS OF CEONP}

Numerous techniques such as hydrothermal, ${ }^{20}$ solvothermal, ${ }^{21,22}$ aqueous precipitation, ${ }^{23}$ reversed micelles, ${ }^{24}$ thermal decomposition $^{25}$ and flame spray ${ }^{26}$ methods have been reported to synthesize CeONP, while maintaining control of its size and properties. The synthesized CeONP can be bare or wrapped with a coating of protective substances that can be hydrophilic ${ }^{20}$ or hydrophobic. ${ }^{22}$ For biological use, biocompatible CeONP has been systematically synthesized in pure water ${ }^{27}$ or with the protection of polyethylene glycol, ${ }^{28}$ dextran, ${ }^{29}$ polyacrylic acid, ${ }^{10}$ cyclodextrin, ${ }^{16}$ glucose $^{17}$ and so on. Synthetic methods are important because they

Division of Biological Inorganic Chemistry, State Key Laboratory of Rare Earth Resource Utilization, Laboratory of Chemical Biology, Changchun Institute of Applied Chemistry, University of Chinese Academy of Sciences, Chinese Academy of Sciences, Changchun, China

Correspondence: Dr X Qu, Division of Biological Inorganic Chemistry, State Key Laboratory of Rare Earth Resource Utilization, Laboratory of Chemical Biology, Changchun Institute of Applied Chemistry, University of Chinese Academy of Sciences, Chinese Academy of Sciences, 5625 Renmin Street, Changchun 130022 , China.

E-mail: xqu@ciac.ac.cn

Received 26 August 2013; revised 13 November 2013; accepted 24 November 2013 
determine the solubility, size, surface condition, charge, structural arrangement and morphology of nanoparticles, thus affecting their properties, including catalytic activities. One major merit of using nanomaterials is that their properties can be controlled and tailored in a predictable manner to meet the needs of specific applications.

\section{THE MULTI-ENZYME MIMETIC ACTIVITIES OF CEONP}

\section{The mechanism of the catalytic activity of CeONP}

Unlike the other lanthanoid series, which usually exhibit a trivalent $(+3)$ state, the cerium atom can exist in either the +3 (fully reduced) or +4 (fully oxidized) state, because it has two partially filled subshells of electrons, $4 f$ and $5 d$, with several excited substates predicted. ${ }^{30}$ In the oxide of cerium, the $\mathrm{Ce}^{4+}$ state with the stable electronic configuration of xenon is preferentially formed. It crystallizes in the fluorite structure in which every cerium atom is surrounded by eight oxygen anions and every oxygen atom occupies a tetrahedral position. Nonetheless, a significant concentration of intrinsic defects is usually present, with a portion of cerium present in the $\mathrm{Ce}^{3+}$ valence state having the deficiency of positive charge compensated by oxygen vacancies. ${ }^{3,30}$ The relative amount of cerium ions $\mathrm{Ce}^{3+}$ and $\mathrm{Ce}^{4+}$ is a function of particle size. ${ }^{31}$ In general, the fraction of $\mathrm{Ce}^{3+}$ ions in the particles increases with decreasing particle size. The techniques used to determine the $\mathrm{Ce}^{3+} / \mathrm{Ce}^{4+}$ ratios include X-ray photoelectron spectroscopy, ${ }^{31} \mathrm{X}$-ray absorption nearedge spectroscopy, ${ }^{32}$ electron magnetic resonance spectroscopy ${ }^{33}$ and UV-visible absorption spectroscopy. ${ }^{34}$

It is remarkable and interesting that CeONP could have a dual role as an oxidation catalyst and reduction catalyst, depending on the reaction conditions. The activities derive from the quick and expedient mutation of the oxidation state between $\mathrm{Ce}^{4+}$ and $\mathrm{Ce}^{3+}$. The cerium atom has the ability to easily and drastically adjust its electronic configuration to best fit its immediate environment. ${ }^{35}$ It also exhibits oxygen vacancies, or defects, in the lattice structure that arise through loss of oxygen and/or its electrons, alternating between $\mathrm{CeO}_{2}$ and $\mathrm{CeO}_{2-\mathrm{x}}$ during redox reactions. The $\mathrm{Ce}^{4+}$ and the low formation energies of surface vacancies are important for oxidation, whereas the $\mathrm{Ce}^{3+}$ and electron shuffling within the lattice oxygen vacancies provide power for reduction. Moreover, the addition or removal of oxygen atoms in the oxidizing or reducing process involves a minimal reorganization of the skeleton arrangement of the cerium atoms and the retention of the fluorite structure. ${ }^{35}$ This structural property facilitates the regenerative ability of CeONP to the initial state and thereby could be recycled to act catalytically.

Strong experimental evidence for the successful shift between $\mathrm{Ce}^{4+}$ and $\mathrm{Ce}^{3+}$ states and the defect structure of CeONP have been provided for the catalytic mechanism. ${ }^{36-39}$ Recently, direct observations of atomic-scale surface structures of CeONP have been realized by noncontact atomic force microscopy ${ }^{40,41}$ and highresolution scanning tunneling microscopy. ${ }^{42}$ The noncontact atomic force microscopy images showed that the hexagonally arranged bright oxygen spots could be observed on the nearly stoichiometric $\mathrm{CeO}_{2}$ (111) surface (Figure 1A), whereas surface oxygen point defects were observed as isolated dark depressions on the reduced $\mathrm{CeO}_{2}$ (111) surface (Figure 1B). When exposed to oxygen atmosphere, the defects could be easily healed (Figure 1C). High-resolution scanning tunneling microscopy studies gave similar results to those of noncontact atomic force microscopy. ${ }^{42}$ The microscopy images provide direct evidence for the dynamic behavior of surface oxygen atoms of CeONP in different oxidation states, which are important for understanding the role of CeONP in catalysts.
Recently, it was found that CeONP could not only be used as an industrial catalyst, but that it also has multi-bioenzyme mimetic properties. ${ }^{10,43-46}$ Although the mechanisms of these activities were not thoroughly studied and fully understood, one could hypothesize that the properties resemble the mechanism of catalytic activities observed in nonbiological systems. These enzyme-mimetic properties make CeONPs useful in biotechnology and therapeutics.

\section{Superoxide dismutase mimetic activity}

Superoxide dismutase $(\mathrm{SOD})^{47}$ is an enzyme that repairs cells and reduces the damage done to them by superoxide, the most common free radical in the body. It catalyzes the disproportionation of superoxide to $\mathrm{H}_{2} \mathrm{O}_{2}$ and $\mathrm{O}_{2}$ :

$$
\begin{aligned}
& \mathrm{M}^{(\mathrm{n}+1)+}-\mathrm{SOD}+\mathrm{O}_{2}^{\bullet-} \rightarrow \mathrm{M}^{\mathrm{n}+}-\mathrm{SOD}+\mathrm{O}_{2} \\
& \mathrm{M}^{\mathrm{n}+}-\mathrm{SOD}+\mathrm{O}_{2}^{\bullet-} \rightarrow \mathrm{M}^{(\mathrm{n}+1)+}-\mathrm{SOD}+\mathrm{H}_{2} \mathrm{O}_{2},
\end{aligned}
$$

where $\mathrm{M}=\mathrm{Cu}(n=1) ; \mathrm{Mn}(n=2) ; \mathrm{Fe}(n=2)$; and $\mathrm{Ni}(n=2)$.

In this reaction, the oxidation state of the metal cation oscillates between $n$ and $n+1$. The $\mathrm{Ce}^{3+} / \mathrm{Ce}^{4+}$ valence switch ability of CeONP makes it possible for CeONP to be a SOD mimic. Self and colleagues $^{43}$ evaluated the ability of CeONP to react with superoxide in vitro for the first time. They tested two preparations of CeONP with different $\mathrm{Ce}^{3+} / \mathrm{Ce}^{4+}$ ratios and found that CeONP with higher ratios of $\mathrm{Ce}^{3+} / \mathrm{Ce}^{4+}$ exhibited a higher SOD-like activity. ${ }^{34,43}$ On the basis of the reaction mechanism that is known for SOD, they proposed that the dismutation of superoxide by CeONP is catalyzed as follows:

$$
\begin{aligned}
& \mathrm{O}_{2}^{\bullet-}+\mathrm{Ce}^{4+} \rightarrow \mathrm{O}_{2}+\mathrm{Ce}^{3+} \\
& \mathrm{O}_{2}^{\bullet-}+\mathrm{Ce}^{3+}+2 \mathrm{H}^{+} \rightarrow \mathrm{H}_{2} \mathrm{O}_{2}+\mathrm{Ce}^{4+} .
\end{aligned}
$$

Celardo et al. ${ }^{9}$ proposed a more exhaustive molecular mechanism in a later review. As illustrated in Figure 2, using (4) as the original state, superoxide can bind to oxygen vacancy sites around two $\mathrm{Ce}^{3+}$ (5). Next, an electron transfers from one $\mathrm{Ce}^{3+}$ to an oxygen atom. Two protons from the solution can bind to the two electronegative oxygen atoms to form one molecule of $\mathrm{H}_{2} \mathrm{O}_{2}$ and be released (6). The remaining oxygen vacancy can provide a binding site for a second superoxide molecule (7). After reaction, a second $\mathrm{H}_{2} \mathrm{O}_{2}$ molecule is released and the original $2 \mathrm{Ce}^{3+}$ will be oxidized to $2 \mathrm{Ce}^{4+}$ (1). However, the reaction did not stop with this step. An oxygen vacancy site on the surface (1) presents a $2 \mathrm{Ce}^{4}+$ binding site for one molecule of $\mathrm{H}_{2} \mathrm{O}_{2}$ (2); hence, the $\mathrm{H}_{2} \mathrm{O}_{2}$ will have the role of a reducing agent. After the release of protons, two electrons will transfer to the two cerium ions to reduce them to $2 \mathrm{Ce}^{3+}$ (3). Next, oxygen is released and the fully reduced oxygen vacancy site returns to the initial state (4). The $\mathrm{H}_{2} \mathrm{O}_{2}$ has a paradoxical effect on CeONP for both oxidation and reduction. However, its structural properties allow CeONP to be restored to its initial state. ${ }^{32}$ The kinetics measured by Seal et al. ${ }^{43}$ showed excellent activity for CeONP (3-5 nm), with a constant catalytic rate that exceeded the one determined for the enzyme SOD.

\section{Catalase mimetic activity}

Catalase $^{48}$ is a protective enzyme found in nearly all living organisms that are exposed to oxygen. It is responsible for the degradation of $\mathrm{H}_{2} \mathrm{O}_{2}$, a powerful and potentially harmful oxidizing agent. Although the complete mechanism of catalase is not currently known, the 

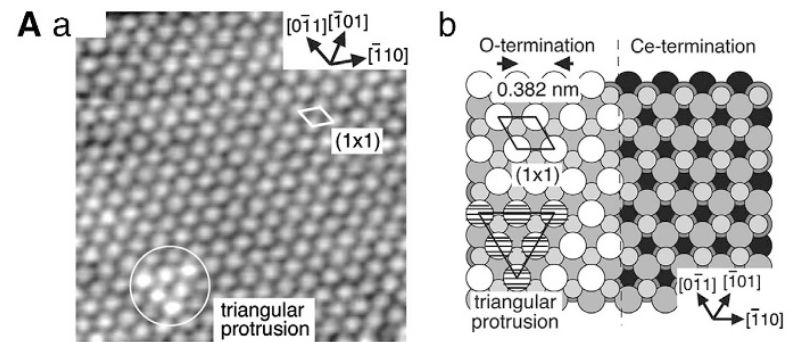

C
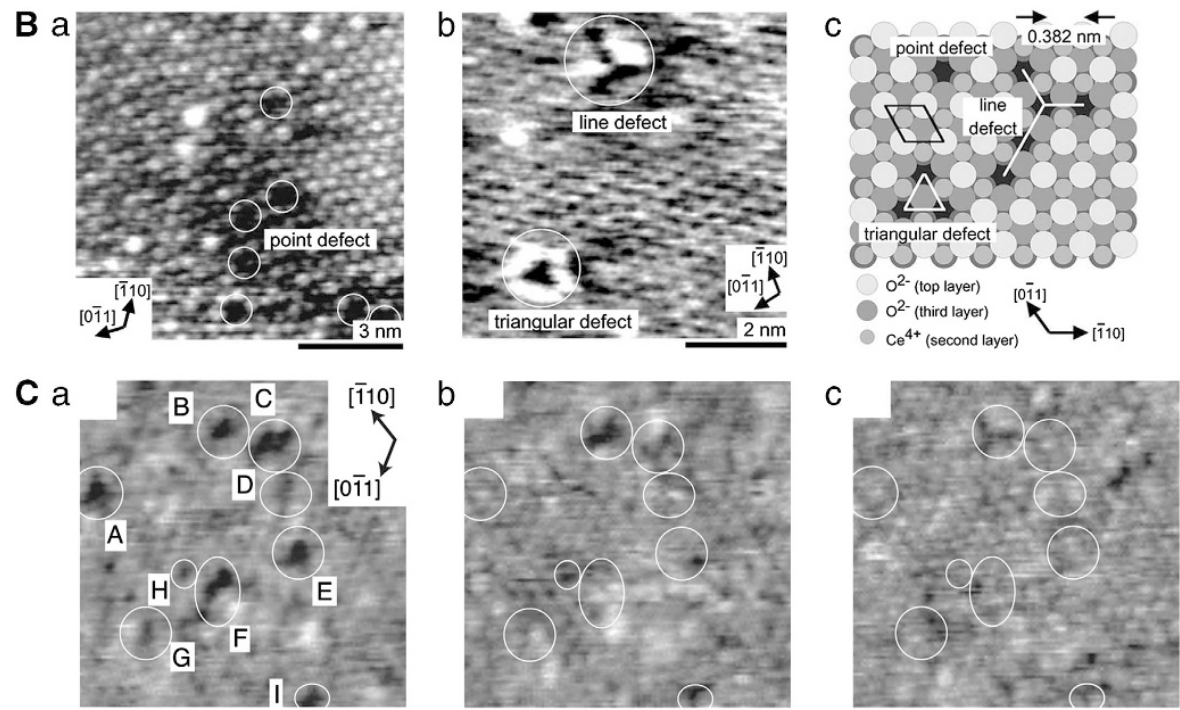

Figure 1 (A) (a) Atom-resolved noncontact atomic force microscopy (NC-AFM) image of nearly stoichiometric $\mathrm{CeO}_{2}$ (111) surfaces. (b and c) Model of the bulk-terminated structure of $\mathrm{CeO}_{2}$ (111) with oxygen-layer termination and cerium-layer termination. ${ }^{41}$ Copyright 2003, American Chemical Society. (B) NCAFM images of slightly reduced $\mathrm{CeO}_{2}$ (111) surface with different densities of oxygen vacancies by different annealing periods: (a) after annealing for $120 \mathrm{~s}$; (b) after annealing for $240 \mathrm{~s}$; and (c) model of multiple defects of line defects and triangular defects observed in b. ${ }^{40}$ Copyright 2002 , Elsevier. (C) Successive NC-AFM images of the same area of a slightly reduced $\mathrm{CeO}_{2}$ (111) surface with multiple defects (a) before and (b and c) after exposure under oxygen exposure at radiation therapy (RT). Multiple defects and point defects were healed by oxygen atoms during the $91 \mathrm{~s}$ taken for scanning. The same positions were indicated by white circles for a guide. ${ }^{41}$ Copyright 2003, American Chemical Society.

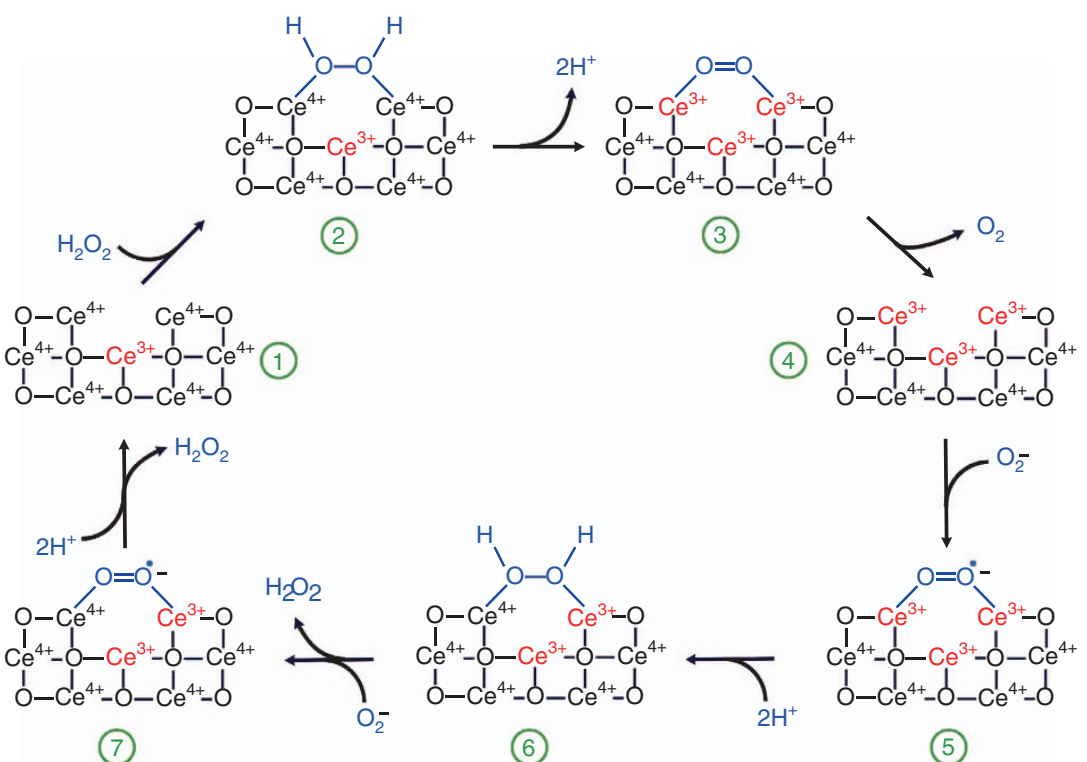

Figure 2 A model of the reaction mechanism for the dismutation of superoxide by cerium oxide nanoparticle (CeONP). ${ }^{9}$ Copyright 2011 , Royal Society of Chemistry. 
reaction is believed to occur in two stages:

$$
\begin{gathered}
\mathrm{H}_{2} \mathrm{O}_{2}+\mathrm{Fe}(\mathrm{III})-\mathrm{E} \rightarrow \mathrm{H}_{2} \mathrm{O}+\mathrm{O}=\mathrm{Fe}(\mathrm{IV})-\mathrm{E}^{\bullet+} \\
\mathrm{H}_{2} \mathrm{O}_{2}+\mathrm{O}=\mathrm{Fe}(\mathrm{IV})-\mathrm{E}^{\bullet} \rightarrow \mathrm{H} 2 \mathrm{O}+\mathrm{Fe}(\mathrm{III})-\mathrm{E}+\mathrm{O}_{2},
\end{gathered}
$$

where $\mathrm{Fe}(\mathrm{III})-\mathrm{E}$ represents the iron center of the heme group attached to the enzyme and $\mathrm{Fe}(\mathrm{IV})-\mathrm{E}^{-}+$is a mesomeric form of $\mathrm{Fe}(\mathrm{V})-\mathrm{E}$.

The catalase-like activity of CeONP was demonstrated by Self and co-workers $^{44}$ via an Amplex Red assay (Invitrogen, Eugene, OR, USA). CeONP could act as catalase mimic in a redox-state-dependent manner, and higher levels of cerium in the +4 state exhibited more significant activity. These findings are of particular interest, as they are in contrast to CeONP's SOD mimetic activity, for which lower +3 / +4 ratios were found to be less efficient. Celardo et al. ${ }^{9}$ proposed a possible molecular mechanism involving two half reactions as illustrated in Figure 3. The oxidative half reaction is shown in Figure 3 (1-4). One molecule of $\mathrm{H}_{2} \mathrm{O}_{2}$ reacts with $\mathrm{Ce}^{4+}$, reducing it to $\mathrm{Ce}^{3+}$ and releasing protons and $\mathrm{O}_{2}$. The reductive half reaction is shown in Figure 3 (4-6-1). A second $\mathrm{H}_{2} \mathrm{O}_{2}$ molecule could bind to the oxygen vacancy site (5), oxidize the $\mathrm{Ce}^{3+}$ back to the initial $\mathrm{Ce}^{4+}$ state and release $\mathrm{H}_{2} \mathrm{O}$. The two processes are analogous to the one found in catalases.

It is worth noting that $\mathrm{H}_{2} \mathrm{O}_{2}$ was produced when CeONP acted as SOD. In vivo, excess $\mathrm{H}_{2} \mathrm{O}_{2}$ is believed to have more toxic potential than superoxide because it is the substrate for the Fenton reaction and generates hydroxyl radicals $(\mathrm{OH} \cdot)$, the most destructive reactive oxygen species (ROS). ${ }^{49}$ Fortunately, CeONP has both catalase- and SOD-like activities. The $\mathrm{H}_{2} \mathrm{O}_{2}$ generated in the SOD-mimetic process can enter into the catalase-mimetic dismutation cycle and produce innocuous $\mathrm{H}_{2} \mathrm{O}$ and $\mathrm{O}_{2}$. This makes CeONP an excellent antioxidant. However, its antioxidative quality is only effective when the two enzyme-like activities of CeONP are coordinated, that is, the $\mathrm{H}_{2} \mathrm{O}_{2}$ decomposition rate should be equal to or greater than its generation rate. According to recent reports, many factors such as particle size, $\mathrm{Ce}^{3+} / \mathrm{Ce}^{4+}$ ratio, ${ }^{32,43,50}$ buffer species ${ }^{51}$ and $\mathrm{pH}$ conditions ${ }^{51,52}$ could affect the enzyme-mimetic properties of CeONP.
The particle size and redox-state-dependent manner have been discussed above. For buffer species, exposure of CeONP to phosphate buffer would result in a decrease in SOD-mimetic activity and an increase in catalase-mimetic activity. ${ }^{51}$ Given that phosphate is a basic substance in biological systems, its impact on the activity of CeONP should be taken into account when CeONP is used in cell culture or animal studies. However, the ability to reverse the 'poisoning' of the SOD-mimetic activity in vivo can be investigated in future studies. The $\mathrm{pH}$ value could also influence the catalytic properties of CeONP. ${ }^{51,52}$ The catalase-mimetic activity of CeONP under a physiological $\mathrm{pH}$ is significantly diminished when under an acidic $\mathrm{pH}$, whereas the SOD activity of CeONP is only slightly affected over a variety of $\mathrm{pH}$ changes. This suggests that at a more acidic $\mathrm{pH}$, CeONP cannot detoxify the hydrogen peroxide at the same rate as it can at neutral $\mathrm{pH}$, whereas the rate for superoxide converting to peroxide is not affected. Thus, in an environment with low $\mathrm{pH}$, CeONP could be harmful.

\section{Hydroxyl radical scavenging}

The hydroxyl radical $(\cdot \mathrm{OH})^{49}$ is one of the strongest oxidants and most biologically active free radicals. There are two methods by which living systems remove hydroxyl radicals: blocking hydroxyl radical initiation by antioxidant enzymes (SOD, glutathione peroxidase and catalase) or breaking the hydroxyl radical chain reaction using nonenzymatic antioxidants. ${ }^{49}$

Hickman and co-workers ${ }^{53}$ found that ultrafine CeONP $(2-5 \mathrm{~nm})$ with mixed valence states had a significant neuroprotective effect on $\mathrm{H}_{2} \mathrm{O}_{2}$-treated adult spinal cord model systems designed to prevent oxidative injury. As $\mathrm{H}_{2} \mathrm{O}_{2}$ provides a source of hydroxyl radicals, which have a major role in oxidative injury, Hickman and coworkers ${ }^{53}$ proposed that the protective effect of CeONP on the spinal cord involved its free-radical scavenging effect. Using $\mathrm{H}_{2} \mathrm{O}_{2}$ to treat CeONP directly, they observed a significant color change from light yellow to deep orange (Figure 4a), which indicates that $\mathrm{Ce}^{3+}$ (colorless) could be used as an antioxidant to react with the free radicals generated from $\mathrm{H}_{2} \mathrm{O}_{2}$ and oxidize to $\mathrm{Ce}^{4+}$ (orange). When the solution was kept in dark conditions for 30 days, the color

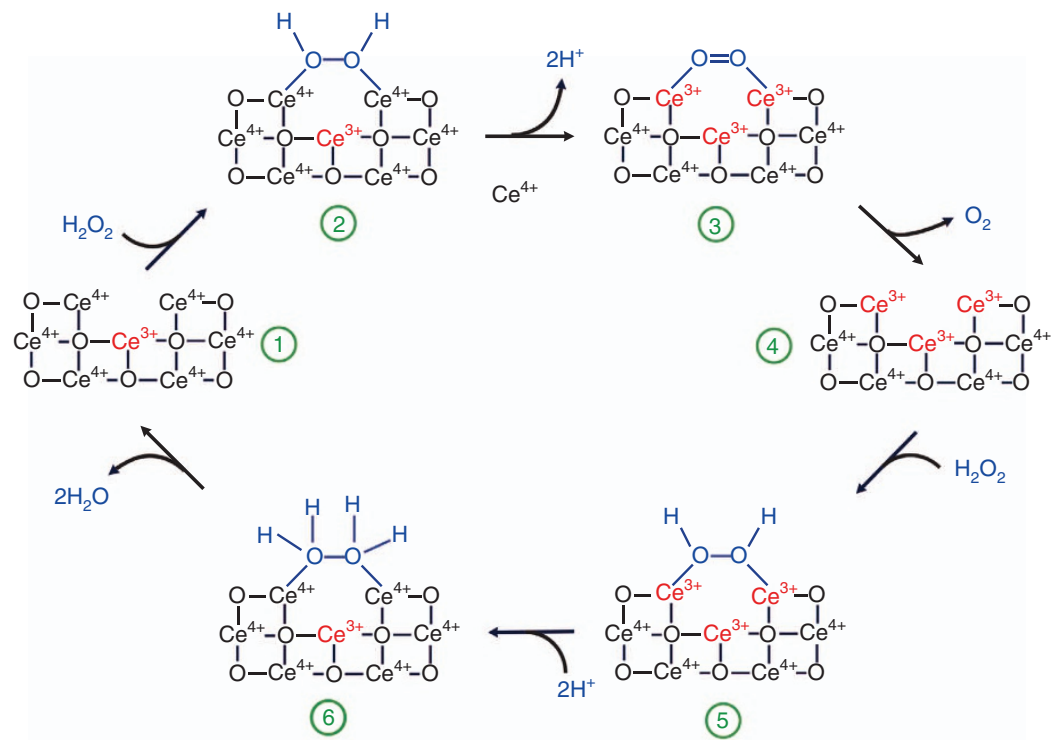

Figure $3 \mathrm{~A}$ model of the reaction mechanism for the complete dismutation of hydrogen peroxide by cerium oxide nanoparticle (CeONP). ${ }^{9}$ Copyright 2011 , Royal Society of Chemistry. 
a
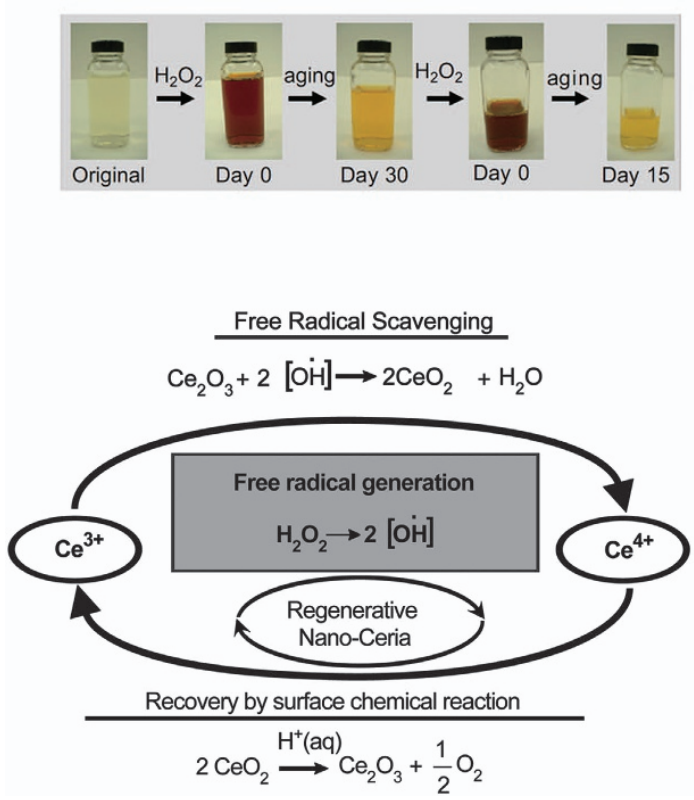

b

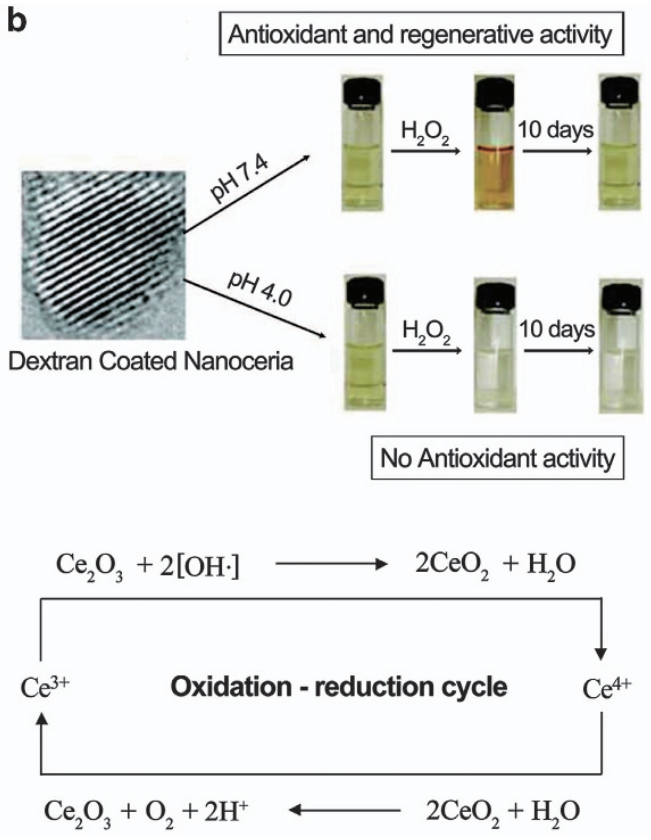

Figure 4 (a) Top: Ppotographs of color changes in solutions of dextran-coated cerium oxide nanoparticle (CeONP) treated with hydrogen peroxide at different time intervals. Below: schematic detailing the proposed regenerative properties of CeONP and probable mechanism of the CeONP free-radical scavenging property and autocatalytic behavior. ${ }^{53}$ Copyright 2007, Elsevier. (b) Top: photographs of color changes in solutions of dextran-coated CeONP at physiological and acidic $\mathrm{pH}$ on addition of hydrogen peroxide. Below: a more detailed probable mechanism of CeONP free-radical scavenging property and autocatalytic behavior. ${ }^{29}$ Copyright 2008, Wiley-VCH.

returned to its original state, which reflected the regeneration of CeONP. Hickman and co-workers ${ }^{53}$ proposed that the autoregenerative property appears to be the key to CeONP's neuroprotective action as an antioxidant (Figure $4 \mathrm{a})$. In a later study, Perez et $a .^{29}$ found that the regenerative ability (or autocatalytic nature) of CeONP was related to the $\mathrm{pH}$ environment (Figure $4 \mathrm{~b}$ ). Under physiological ( $\mathrm{pH}$ 7.4) and basic conditions, the autocatalytic property remained, whereas under acidic conditions, the property was not observed, even when the $\mathrm{pH}$ was raised to 7.4. Thus, they revised the reaction equation to make it more reasonable (Figure $4 \mathrm{~b}$ ).

The hydroxyl radical scavenging ability of CeONP elaborated above was inferred from its antioxidant properties. Recently, Xue et al. ${ }^{50}$ provided direct evidence for the hydroxyl radical scavenging activity of CeONP via a methyl violet assay. They further demonstrated that CeONP could only partly, not completely, eliminate the generated hydroxyl radicals. The activity was also shown to be size dependent and was believed to have a close correlation with $\mathrm{Ce}^{3+}$ at the surface of the particles.

\section{Nitric oxide radical scavenging}

The nitric oxide radical $(\cdot \mathrm{NO})^{54}$ is a gaseous free radical that exhibits multifaceted biological effects, both beneficial and damaging. NO can react rapidly with the superoxide radical, forming the highly reactive peroxynitrite anion $\left(\mathrm{ONOO}^{-}\right)$, a more powerful and toxic oxidant. A number of disease states are characterized by abnormally high $\cdot$ NO production, and removing excess - NO could have salutary effects.

Inspired by CeONP's NO exhaust gas treatment ability and $\cdot$ NO adsorption and decomposing ability in industry, ${ }^{55}$ Self and coworkers ${ }^{56}$ explored and demonstrated the $\cdot$ NO scavenging ability of CeONP under physiologically relevant conditions. Surprisingly, compared with the superoxide scavenging properties that prefer a higher level of cerium in the $\mathrm{Ce}^{3+}$ state, the $\cdot \mathrm{NO}$ scavenging ability is present in CeONP with a lower $\mathrm{Ce}^{3+} / \mathrm{Ce}^{4+}$ ratio. Similar to the $\mathrm{NO}$ dismutation mechanism of iron porphyrins, ${ }^{57}$ a possible mechanism by which CeONP scavenges NO through formation of an electropositive nitrosyl ligand caused by internal electron transfer from $\mathrm{NO}$ to a $\mathrm{Ce}^{4+}$ site was proposed:

$$
\mathrm{Ce}^{4+}+\bullet \mathrm{NO} \rightarrow\left[\mathrm{Ce}^{4+} \mathrm{NO} \leftrightarrow \mathrm{Ce}^{3+} \mathrm{NO}^{+}\right]
$$

\section{Peroxidase mimetic activity}

Peroxidases ${ }^{58}$ are a class of oxidoreductases that are widely distributed among living organisms. They are able to catalyze the reduction of peroxide and oxidize various substrates as follows:

$$
\mathrm{ROOR}^{\prime}+\text { electrondonor }\left(2 \mathrm{e}^{-}\right)+2 \mathrm{H}^{+} \rightarrow \mathrm{ROH}+\mathrm{R}^{\prime} \mathrm{OH} \text {. }
$$

This biochemical function confers on them a role in many different and important biological processes, such as defense mechanisms, immune responses and pathogeny. Peroxidases also occupy a prominent position in biotechnology..$^{58}$

Recently, many nanomaterials were found to have peroxidase-like activity and the potential to replace nature peroxidase by virtue of their stability, low cost and obtainability. ${ }^{59,60}$ For transition metal oxide material-based peroxidase mimics, their activities were mostly derived from the metal ions that have a catalytic activity using $\mathrm{H}_{2} \mathrm{O}_{2}$ as substrate through a mechanism similar to that of the Fenton reaction. ${ }^{59}$ Self and co-workers ${ }^{61}$ found that cerium ions could also perform a Fenton-like reaction:

$$
\begin{aligned}
& \mathrm{Ce}^{3+}+\mathrm{H}_{2} \mathrm{O}_{2}+\mathrm{H}^{+} \rightarrow \mathrm{Ce}^{4+}+\mathrm{OH} \bullet+\mathrm{H}_{2} \mathrm{O} \\
& \mathrm{OH} \bullet+\mathrm{H}_{2} \mathrm{O}_{2} \rightarrow \mathrm{HO}_{2}^{-}+\mathrm{H}_{2} \mathrm{O} \\
& \mathrm{Ce}^{4+}+\mathrm{HO}_{2}^{-} \rightarrow \mathrm{O}_{2}+\mathrm{Ce}^{3+}+\mathrm{H}^{+}
\end{aligned}
$$

This finding gives cerium-containing materials the potential to be used as peroxidase mimics. In a later report, $\mathrm{Lv}$ and colleagues ${ }^{45}$ 
demonstrated directly that CeONP synthesized by a simple hydrothermal method had a peroxidase-like activity. Furthermore, based on this property the group developed a facile colorimetric method for glucose detection. ${ }^{45}$ The peroxidase-like activity provides CeONP broad potential applications in environmental chemistry, biotechnology and medicine.

\section{Oxidase mimetic activity}

An oxidase ${ }^{62}$ is an enzyme that catalyzes an oxidation-reduction reaction involving molecular oxygen $\left(\mathrm{O}_{2}\right)$ as the electron acceptor. The importance of oxidases is emphasized by their multiple physiological roles. Studies by Perez and colleagues ${ }^{10}$ revealed that CeONP synthesized by an in situ aqueous-phase method showed oxidase-like activities, because they could quickly oxidize several organic substrates without oxidizing agents. In a later study, Gao and colleagues ${ }^{63}$ proposed that instead of being postulated as oxidase mimic, CeONP was a plain nanoparticulate oxidant and dissolved completely after being reduced under acidic conditions.

Despite these arguments, CeONP used as oxidation catalyst has been widely exploited and has important roles in many fields. For example, in three-way catalysis, ${ }^{7,64} \mathrm{CeONP}$ catalyzes the conversion of the hydrocarbons, $\mathrm{CO}$ and $\mathrm{NO}_{\mathrm{x}}{ }^{7,64}$ which includes three simultaneous processes:

$$
\begin{aligned}
& 2 \mathrm{NO}_{\mathrm{x}} \rightarrow \mathrm{xO}_{2}+\mathrm{N}_{2} \\
& 2 \mathrm{CO}+\mathrm{O}_{2} \rightarrow 2 \mathrm{CO}_{2} \\
& \mathrm{C}_{\mathrm{x}} \mathrm{H}_{2 \mathrm{x}+2}+[(3 \mathrm{x}+1) / 2] \mathrm{O}_{2} \rightarrow \mathrm{xCO}_{2}+(\mathrm{x}+1) \mathrm{H}_{2} \mathrm{O} .
\end{aligned}
$$

In the $\mathrm{CO}$ and $\mathrm{C}_{\mathrm{x}} \mathrm{H}_{2 \mathrm{x}+2}$ oxidation reactions, $\mathrm{CeONP}$ has a role similar to oxidase. Regarding the issue of whether CeONP acts directly as an oxidase or an oxidant in organic reagent oxidation, we propose that the role CeONP has may depend on the $\mathrm{pH}$ environment. As previously mentioned, under neutral and basic conditions, the $\mathrm{Ce}^{4+} / \mathrm{Ce}^{3+}$ recycling ability was maintained, thus providing CeONP catalytic activity, whereas under acidic conditions the recycling ability was not observed. Furthermore, $\mathrm{H}^{+}$in the acidic solution can react with CeONP to produce dissoluble cerium ions and $\mathrm{H}_{2} \mathrm{O}$. Therefore, we deduced that under acidic conditions CeONP acts as a consumable oxidant, whereas under neutral or basic conditions it acts as an oxidase because of the regenerative capacity of the $\mathrm{Ce}^{4+}$ oxidative state. The antioxidant and oxidase activities were not contradictory; rather, they were dependent on the related redox potential of the substrate.

\section{Phosphatase-mimetic activity}

A phosphatase ${ }^{65}$ is an enzyme that removes phosphate groups from their substrates by hydrolyzing phosphoric acid monoesters into phosphate ions. Phosphates have important roles in many biological processes, including signal transduction and cell proliferation, differentiation, metabolism and communication. Some metal ions and their complexes were reported to be used as artificial phosphoesterases ${ }^{66}$ that can accelerate the rate of phosphate ester hydrolysis in several ways, including Lewis acid activation, nucleophile activation and leaving group activation. As Lewis acid, some lanthanide ions and their complexes have been shown to be particularly reactive for hydrolyzing phosphate esters. ${ }^{66,67}$ Cerium, the only lanthanide that is easily oxidized to the tetravalent state, has received great attention. ${ }^{67}$

Recent studies have shown that CeONP can hydrolyze phosphate ester bonds of many biologically relevant molecules. ${ }^{46,68,69}$ Qian and colleagues ${ }^{68}$ found that CeONP could efficiently mediate the dephosphorylation of phosphopeptides. As CeONP is a strong Lewis acid, the dephosphorylation effect can be mainly attributed to Lewis acid activation via coordination of phosphoryl oxygen to $\mathrm{Ce}^{4+}$ and nucleophile activation via coordination of hydroxyl to $\mathrm{Ce}^{4+}$. In addition, CeONP can be regarded as a multinuclear metal-oxygen complex, producing a multiple synergistic activation effect and achieving high catalytic activity. Qian and colleagues ${ }^{68}$ also proposed several advantages of using CeONP to mediate the dephosphorylation of phosphopeptides. First, it is safe and simple, and can be removed by centrifugation after treatment. Second, its catalytic activity is high. The dephosphorylation of all model phosphopeptides was completed in $10 \mathrm{~min}$. Third, temperature has little effect on the dephosphorylation; hence, precise control of the treatment temperature is not required, which facilitates the treatment process. ${ }^{68}$

In a later study, Kuchma et al. ${ }^{34}$ reported that many biologically relevant molecules with phosphate ester, excluding DNA, could be hydrolyzed by CeONP. Interestingly, they observed that unlike the single nuclear complexes of cerium, the dephosphorylative activity of CeONP depends on the presence of $\mathrm{Ce}^{3+}$ sites and is inhibited when $\mathrm{Ce}^{3+}$ is converted to $\mathrm{Ce}^{4+}$. This is contrary to the opinions of Qian and colleagues ${ }^{68}$ who proposed a $\mathrm{Ce}^{4+}$-mediated hydrolysis process. To fully understand the mechanism, additional research must be carried out.

\section{BIOLOGICAL APPLICATIONS \\ Bioanalysis}

Nanotechnology has already greatly impacted bioanalysis. ${ }^{70}$ The stable physical and chemical properties make inorganic nanoparticles suitable for use in biological assays to eliminate the shortcomings of organic fluorophores, radioactive labeling or natural enzymes, which are photobleachable, toxic, and expensive and easily degradable, respectively. ${ }^{70}$ These multi-enzyme-like properties have been successfully used for biological detection and analysis.

Oxidase-like activity. On the basis of the oxidase-like activity of CeONP, which facilitated the oxidation of organic molecules to yield chromogenic products, Perez and colleagues ${ }^{10}$ developed robust and reliable colorimetric immunoassays (Figure 5A). They attached folic acid to polyacrylic acid-coated CeONP (PNC) via click chemistry. As folic acid can specifically recognize certain tumor cells with elevated expression of folate receptors on the surface, it could be used instead of the antifolate receptor antibody traditionally used in enzymelinked immunosorbent assay (ELISA). Target cancer cells (such as lung carcinoma cell line, A-549) would capture the folic-acidmodified PNC. Using 3,3',5,5'-tetramethylbenzidine as the reporting substrate, a colorimetric assay could be established. The efficiency was judged by the increase in absorbance at $652 \mathrm{~nm}$ with increasing amounts of A-549 cells. Using cardiac myocytes (H9c2) cells, which have no folate receptors, as a control, the results showed negligible absorbance changes and thus demonstrated selectivity. The device performed best under acidic conditions. A traditional ELISA assay was also carried out using horseradish peroxidase-labeled secondary antibodies to assess the binding of a specific primary antibody to a particular target or surface receptor and evaluated by catalyzing the oxidation of $3,3^{\prime}, 5,5^{\prime}$-tetramethylbenzidine by $\mathrm{H}_{2} \mathrm{O}_{2}$. Perez and colleagues $^{10}$ compared CeONP-based ELISA assay with the traditional ELISA assay and found that the CeONP had several advantages: (1) both the antibody and natural enzyme (horseradish peroxidase) used in traditional ELISA assay were unstable and easy to denature and inactivate, whereas the folic acid and nanoenzyme 
A

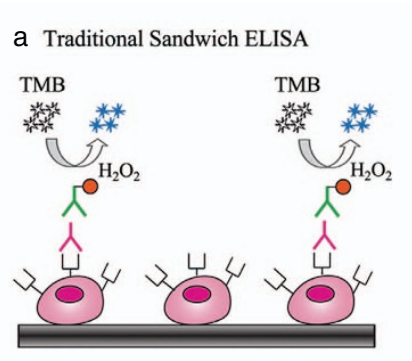

「 HRP Antibody

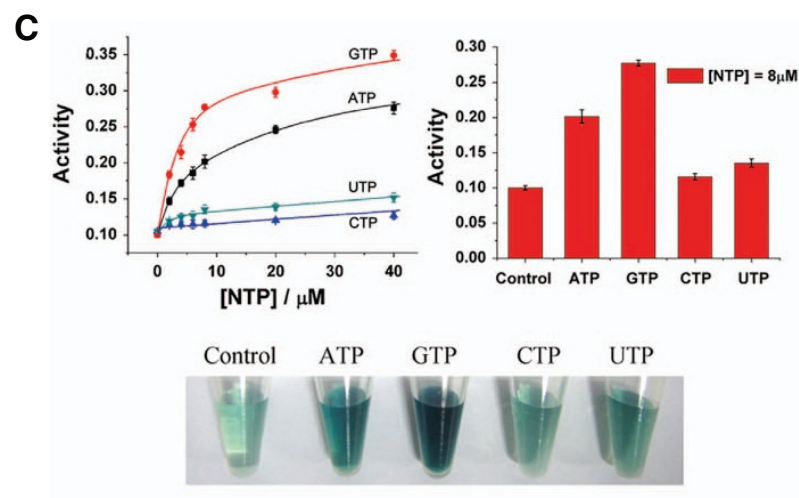

b Nanoceria Based-Sandwich ELSA
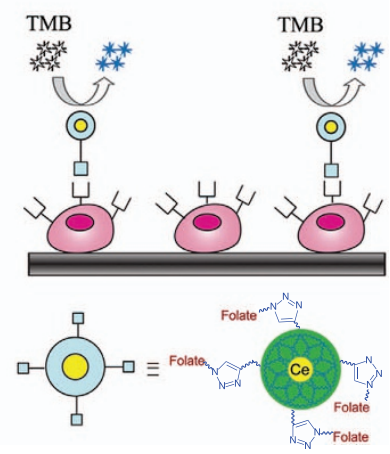<smiles>Nc1nc2[nH]c[nH+]c2c(=O)[nH]1</smiles><smiles>Cc1c[nH]c(=O)[nH]c1=O</smiles>

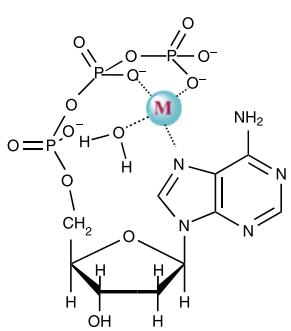

B

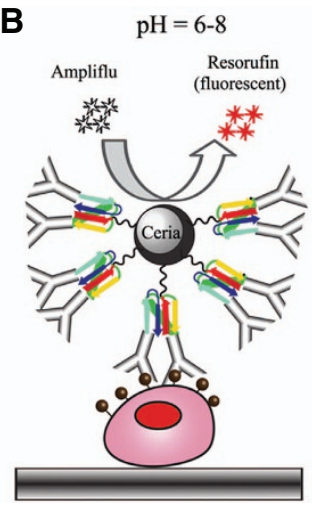

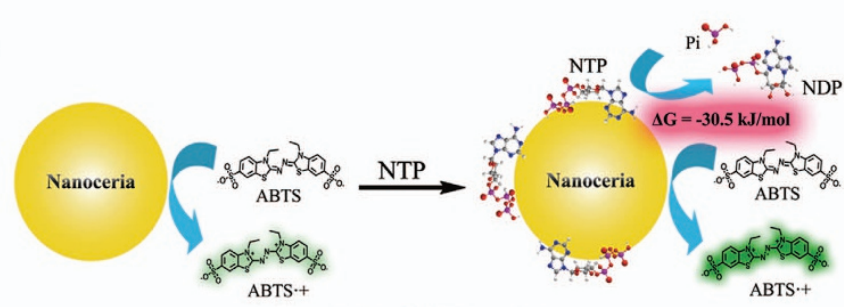

NTPs $+\bigcirc$ SNP typing
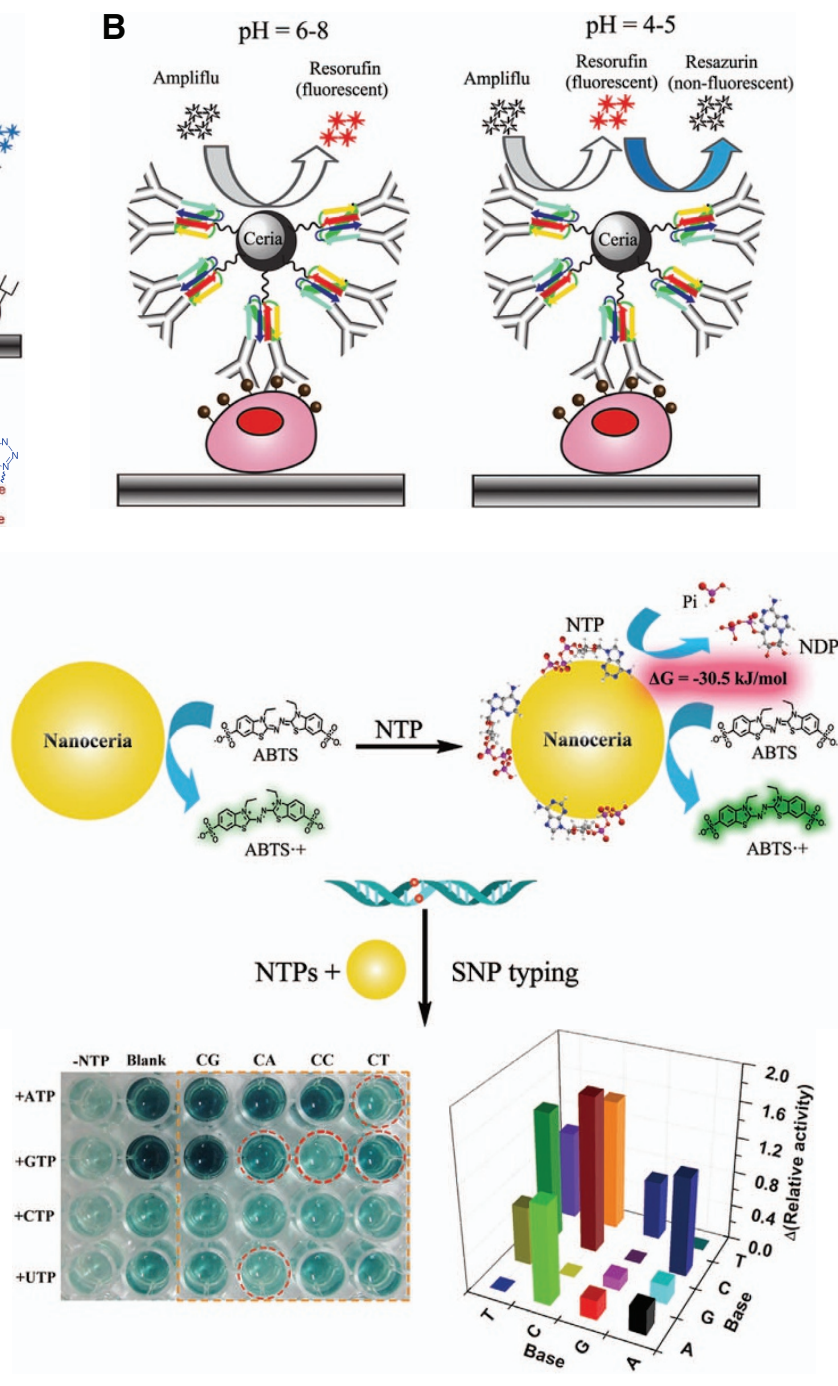

Figure 5 (A) Comparison of (a) traditional enzyme-linked immunosorbent assay (ELISA) and (b) cerium oxide nanoparticle (CeONP)-based ELISA assay. ${ }^{10}$ Copyright 2009, Wiley-VCH. (B) CeONP-based ELISA assay operated in neutral condition. ${ }^{11}$ Copyright 2011, American Chemical Society. (C) The improvement effects of nucleoside triphosphates (NTPS) to the oxidase-like activity of CeONP (left) and the single-nucleotide polymorphism (SNP) typing assays based on this (right). ${ }^{71}$ Copyright 2013, Wiley-VCH.

CeONP used in CeONP-based ELISA assay were more stable; (2) the $\mathrm{H}_{2} \mathrm{O}_{2}$ used in traditional ELISA assay for horseradish peroxidase can easily decompose and lose its oxidative ability, whereas CeONP could oxidize the substrate without $\mathrm{H}_{2} \mathrm{O}_{2}$, thus eliminating the problem; and (3) the cost of the CeONP method is much lower and the operation is much easier, making it more robust than the traditional ELISA assay.

As the CeONP-based ELISA assay described above is optimal under acidic conditions, which limits the use of antibodies and other $\mathrm{pH}$ labile biomolecules as targeting ligands, Perez and colleagues ${ }^{11}$ improved it by exploiting a milder method that could be used under neutral conditions (Figure 5B). They used the fluorescent molecule Ampliflu as the reporter molecule instead of $3,3^{\prime}, 5,5^{\prime}$ tetramethylbenzidine. Ampliflu could be oxidized to fluorescent resorufin by weak oxidase activity, whereas the strong oxidase activity of CeONP would trigger complete oxidation to the nonfluorescent resazurin. As the oxidase-like activity of $\mathrm{CeONP}$ is $\mathrm{pH}$ dependent and has a mild activity at neutral $\mathrm{pH}, \mathrm{CeONP}$ could facilitate the partial oxidation of Ampliflu to the fluorescent resorufin. The method was also used to detect folate-receptor-expressed cancer cells using protein $\mathrm{G}$ and antifolate-receptor antibody co-modified CeONP. The assay is faster and cheaper than traditional ELISA assay and is expected to be used in the clinic.

The combination of oxidase-like and phosphatase-like activities. $\mathrm{Qu}$ and colleagues ${ }^{71}$ reported that nucleoside triphosphates (NTPs) can improve the oxidase-like activity of CeONP and that the enhancement is correlated with the type of NTPs (Figure 5C). This effect was demonstrated to be a result of the coupling of the oxidative reaction with the NTPs' hydrolysis reactions, because CeONP has both oxidase-like and phosphatase-like activities. Unlike when NTPs, acting as coenzymes for biological enzymes, undergo hydrolysis assisted by metal ions as catalysts, in this reaction the hydrolysis of the NTPs could be catalyzed in situ by CeONP, which is attributed to its inherent phosphatase-like activity. The energies released from the NTP hydrolysis can further improve the oxidase-like activity of 
CeONP. Moreover, the catalytic ability of CeONP in NTP hydrolysis is correlated with the type of NTP, which then leads to different improving efficiencies of NTP for the oxidase activity of CeONP. On the basis of the NTP-promoted oxidase-like activity of CeONP and the differences among the various NTPs, Perez and colleagues ${ }^{11}$ developed series-effective colorimetric assays for genotyping (identification of the types of mismatched bases) of singlenucleotide polymorphisms.

pH-dependent bioenzyme mimetic activities. Perez and colleagues ${ }^{13}$ also reported a CeONP-based device for the detection of chronic inflammation by optical and magnetic resonance imaging. Chronic inflammatory conditions are usually associated with high local concentrations of ROS (for example, $\mathrm{H}_{2} \mathrm{O}_{2}$ ) and decreases in $\mathrm{pH}$, which could be used as pro-inflammatory markers. Taking advantage of the pH-dependent antioxidant activities, Perez's group used CeONP as a sensor for ROS and $\mathrm{pH}$ conditions, and visualized these environmental alterations using multimodal iron oxide nanoparticles. They co-encased polyacrylic acid-coated CeONP (PNC) and near-infrared light-emitting carbocyanine DilC18(7)encapsulating dextran-coated iron oxide nanoparticles in a fabricated device (Figure 6A). Under physiological conditions, CeONP could protect the DilC18(7) from fluorescence quenching caused by traces of extracellular ROS. However, in conditions simulating chronic inflammation where the levels of ROS are high and the extracellular milieu's $\mathrm{pH}$ drops, CeONP was not able to protect the carbocyanine DilC18(7) from oxidation-induced quenching, resulting in marked decreases in the device's fluorescence emission. However, the magnetic resonance imaging signal (T2) of iron oxide nanoparticles increased, which was caused by the ROSinduced polymerization of the nanoparticles' dextran coating under low $\mathrm{pH}$ values being catalyzed by the particles themselves. The device was able to distinguish physiological from abnormal ROS and $\mathrm{pH}$ levels (Figure 6A); thus, it could have applications for a broad range of diseases that have a proinflammatory component.

Peroxidase-like activity. Li et al. ${ }^{12}$ found that PNC can facilitate the fast oxidation of luminol by $\mathrm{H}_{2} \mathrm{O}_{2}$ and greatly enhance the chemiluminescence intensity of this system. On the basis of this, they designed a sandwich method for detection of human thrombin. They immobilized the thrombin aptamer onto a magnetic bead for specific thrombin capture. Au nanoparticles containing bio-bar-coded PNC and reporter DNA complementary to thrombin aptamer were used as amplified labels, and luminol- $\mathrm{H}_{2} \mathrm{O}_{2}-\mathrm{PNC}$ was employed as a reporter system. The use of $\mathrm{PNC}$ for the direct catalysis of the luminol- $\mathrm{H}_{2} \mathrm{O}_{2}$ system avoided poisoning by $\mathrm{HBr} / \mathrm{Br}_{2}$ or $\mathrm{HNO}_{3}$ solution, which are generally used in other analysis. The PNC's ability to act as catalyst in the luminol- $\mathrm{H}_{2} \mathrm{O}_{2}$ system contributed to its peroxidase-like activity.

Colorimetric property. As well as catalyzing the colorimetric reactions of organic dyes, CeONP could also be used as a colorimetric agent itself by virtue of its color-changing ability corresponding with a shift in redox state. ${ }^{29,53}$ Taking advantage of this, Andreescu and
A
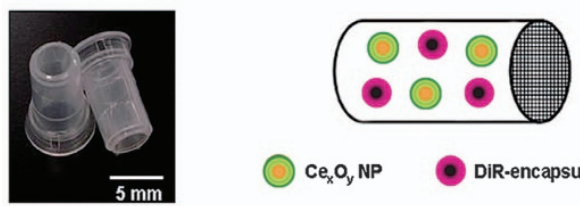

$\mathrm{Ce}_{x} \mathrm{O}_{y} \mathrm{NP}$
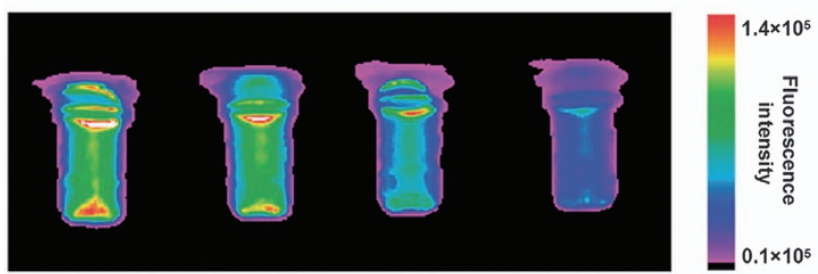

$\underset{(\mathrm{pH} \mathrm{7})}{\mathrm{NM} \mathrm{H} \mathrm{H}_{2} \mathrm{O}}$ $\underset{(\mathrm{pH} 7)}{\mathrm{M} \mathrm{H}_{2}}$ $\mu \mathrm{M} \mathrm{H}_{2} \mathrm{O}_{2}$ $6 \mu \mathrm{M} \mathrm{H}_{2} \mathrm{O}_{2}$ (pH 6)
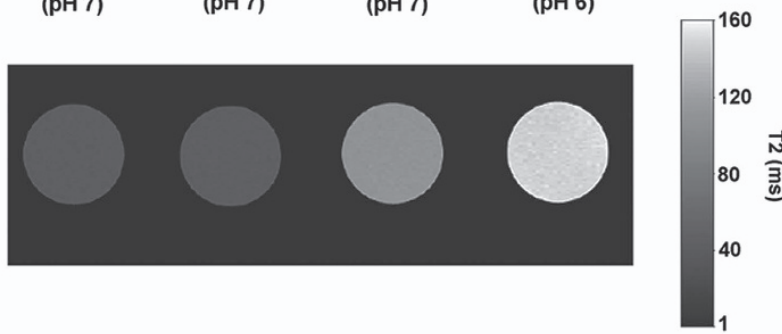

B
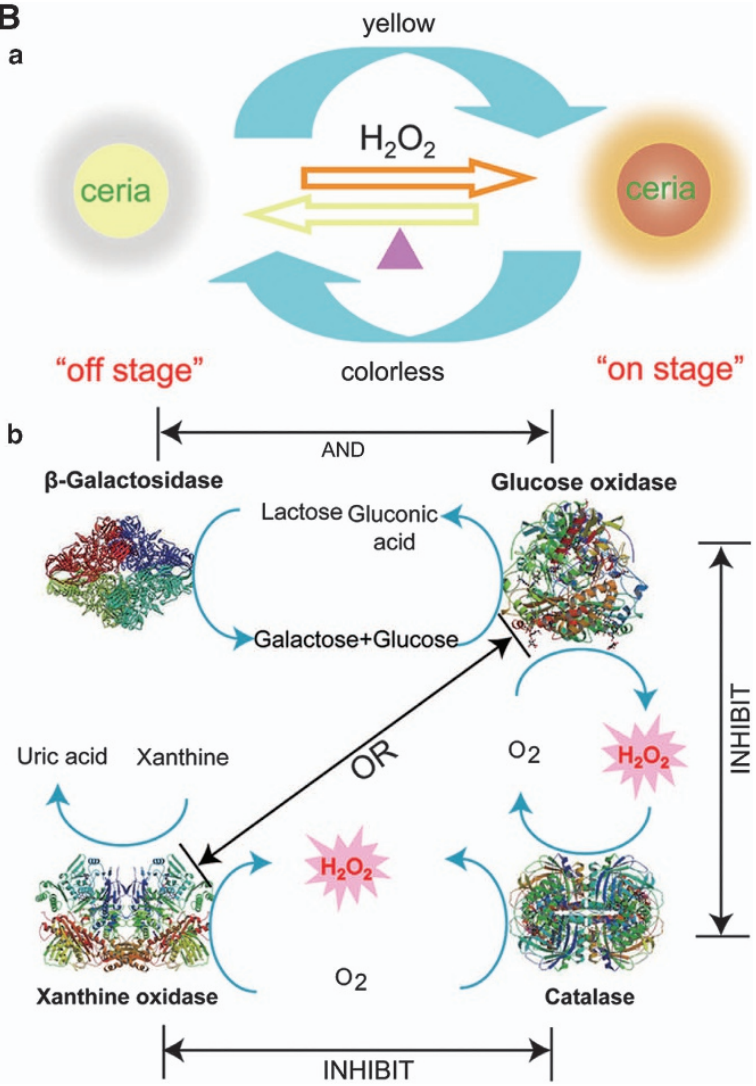

Figure 6 (A) Photograph of cerium oxide nanoparticle (CeONP)-based chronic inflammation detection device and images of the changes in fluorescence emission and magnetic resonance imaging (MRI) signal (T2) under different clinical conditions. ${ }^{13}$ Copyright 2012 , Royal Society of Chemistry. (B) Illustration of thermally regenerable CeONP-based logic gate device. (a) Thermally responsive switch based on CeONP. (b) The operation of logic gates based on biocatalytic reactions. ${ }^{15}$ Copyright 2012, Wiley-VCH. 
colleagues $^{14}$ described a novel concept using CeONP as a chromogenic component for colorimetric paper bioassays. The core principle of the bioassay was an $\mathrm{H}_{2} \mathrm{O}_{2}$-induced redox state shift of surface cerium ions from $\mathrm{Ce}^{3+}$ to $\mathrm{Ce}^{4+}$ species in $\mathrm{CeONP}$ that was accompanied by visible color changes from white-yellowish to dark orange. ${ }^{29,53}$ Thus, it could be used for $\mathrm{H}_{2} \mathrm{O}_{2}$-related molecular analysis. Using glucose as a model analyte, Andreescu and colleagues $^{14}$ co-immobilized CeONP and glucose oxidase onto filter paper by a silanization procedure to construct a glucose-sensing paper. The assay is fully reversible and can be reused for multiple cycles. The reversible process takes place gradually over several days at room temperature or within several minutes by slight heating. The study provides a new nanoparticle platform for the fabrication of paper-based colorimetric bioassays.

On the basis of the $\mathrm{H}_{2} \mathrm{O}_{2}$-induced color changing and regenerative capability of CeONP, Lin et al. ${ }^{15}$ constructed a novel resettable and colorimetric logic network by using CeONP as a signal transducer (Figure 6B). The CeONP solution is nearly colorless, whereas the addition of $\mathrm{H}_{2} \mathrm{O}_{2}$ causes an instantaneous color change to yellow. They defined the yellow solution as ON state (1) and the colorless solution as OFF state (0). As $\mathrm{H}_{2} \mathrm{O}_{2}$ is a common product of oxidative metabolism in living organisms, it can be generated by various types of enzymatic cascade cycles. Thus, Lin et al. ${ }^{15}$ combined the $\mathrm{H}_{2} \mathrm{O}_{2}$ responsive $\mathrm{CeONP}$ and biocatalytic reactions to construct robust logic gates (Figure 6B). They designed multiple input signal systems and demonstrated that these systems could be scaled up to perform large network-mimicking natural biochemical pathways. The regenerative capability of CeONP made the logic gates feasible to reset to their initial conditions by heating. This CeONP-based logic gate solved the two major problems of chemical computing systems ${ }^{72}$ : (1) difficult to scale up for assembling large networking systems because of the interference between reactions and the incompatibility of various chemical gates operating under different conditions; (2) unable to realize the logic system reset, which is a crucial property for practical applications. Finally, Lin et al. ${ }^{15}$ applied the proposed system in practical logic sensing. Compared with traditional sensing devices, logic biosensors are able to intelligently analyze the relationship between different targets. This logic system has potential applications in the field of intelligent diagnostics.

\section{Diseases}

Cerium compound applications in biomedicine. The pharmacological properties of cerium were discovered more than a century ago and have been used to treat many types of diseases. ${ }^{73}$ Cerium(III) oxalate has been widely used to treat vomiting-related diseases such as pregnancy, sea-sickness, chronic diarrhea and neurological disorders, including epilepsy and chorea. ${ }^{74}$ Cerium nitrate is available as an adjunct to silver sulfadiazine cream for the topical treatment of extensive burns. ${ }^{75}$ According to clinical reports from the pioneering era of cancer chemotherapy, cerium(III) iodide could cause tumor shrinkage and improved quality of life. ${ }^{76}$ These diverse biomedical effects contributed to the biorelated properties of cerium, such as a resemblance to calcium, bacteriostatic and bactericidal activities, and immunomodulatory properties. ${ }^{73}$

Although the cerium compounds have the potential to be used in biomedicine, there are many drawbacks: (1) small molecule drugs cannot be easily taken up directly by cells; (2) the short blood circulation time and nonspecific biodistribution of cerium compounds would cause many unwanted side effects; and (3) many cerium compounds, such as cerium oxalate, are nearly insoluble in water, which makes their absorption by organisms difficult.
Nanostructured materials can be employed to overcome these limitations, because they can be taken up by mammalian cells easily through many pathways. Being easy to modify and having long blood circulation times make nanostructured materials potentially targetable with a controllable distribution in vivo. In addition, the insolubility could be easily solved by wrapping the material in a layer of watersoluble polymers. The pharmacological potentials of CeONP for many diseases has increased in recent years. ${ }^{9,77}$

Oxidative stress-related diseases. Oxidative stress $^{78}$ in biological systems results from an imbalance between ROS and nitrogen species production and antioxidant levels. ROS and reactive nitrogen species are potent oxidizing and nitrating agents that include $\mathrm{O}_{2} \cdot-, \cdot \mathrm{HO}, \mathrm{H}_{2} \mathrm{O}_{2}, \cdot \mathrm{NO}$ and $\mathrm{ONOO}^{-}$. These reactive species are normal byproducts and have dual roles in the organism, causing toxicity or acting as signaling molecules, depending on concentration, location and intracellular conditions. ${ }^{78}$ In normal biological systems, when excess reactive species are produced to a limited degree, cells are able to counteract the damage through innate mechanisms, including enzyme antioxidants and endogenous reductants. However, when the production of reactive species exceeds the capacity of cellular defense systems or when cell defense systems are compromised by aging or diseases, problems can arise. Such aberrant active species can cause substantial damage, or oxidative stress, to molecular structures in biological organisms. Oxidative stress has a role in aging and in a variety of human disease states, such as inflammatory and autoimmune diseases, arthritis, cardiovascular disease and neurodegenerative disorders.

Because of its multiple antioxidant-enzyme-like activities, including SOD, catalase, peroxidase-like activities, and hydroxyl radical and nitric oxide radical scavenging properties, CeONP was expected to scavenge almost all types of reactive species. This makes it superior to any antioxidant enzyme or molecule because they often scavenge only a single type of free radical before being inactivated. Recently, CeONP has been receiving much attention as a potential antioxidant agent in vivo and as a potential therapeutic tool in the treatment of oxidative-stress-related diseases.

Neurodegenerative disorders. Being the most active organ in the body, the brain and central nervous system are particularly susceptible to oxidative stress because of high oxygen utilization, low levels of endogenous antioxidant systems and high levels of polyunsaturated fatty acids, which are subject to lipid peroxidation. ${ }^{79}$ Increased oxidative stress and free radical production are associated with several neurodegenerative diseases, such as trauma, Alzheimer's disease $(\mathrm{AD})$ and Parkinson's diseases, ischemic stroke and aging. ${ }^{80}$ Therapeutic efforts aimed at the removal of ROS or prevention of their formation are considered to be beneficial in neurodegenerative diseases. However, use of molecular antioxidants in abolishing these pathological conditions has thus far met with only limited success, which was attributed to the limits of molecular antioxidants: (1) they are not easily taken up directly by cells, which results in an inability to achieve satisfactory levels of antioxidants at the site of injury; (2) many can scavenge only one type of reactive species, whereas diseases often generate mixed reactive species of more than one type; and (3) they cannot cross the blood-brain barrier (BBB). ${ }^{81}$ Therefore, there is an urgent need to design novel classes of antioxidants that are superior to molecular antioxidants. Special attention has been paid to CeONP as an antioxidant, because it can scavenge almost all types of reactive species, has excellent regeneration ability and can cross the BBB because of its nano size. ${ }^{82}$ 
Recently, CeONP has been demonstrated to successfully protect neurons from free-radical-mediated damage initiated by UV light, $\mathrm{H}_{2} \mathrm{O}_{2}$, irradiation and excitotoxicity. ${ }^{83-86}$ Schubert et al. ${ }^{85}$ reported protection from exogenous oxidants in a neuronal cell line (HT22) in the presence of CeONP and yttrium oxide nanoparticles. Das and colleagues $^{86}$ reported that CeONP provides a significant neuroprotective effect on adult rat spinal cords against $\mathrm{H}_{2} \mathrm{O}_{2}$. CeONP can also prolong the lifespan of healthy cortical brain cells by about up to sixfold. ${ }^{83,84}$ Inspired by these neutron-protective effects, the application of CeONP for treatment of neurodegenerative disorder-related diseases was studied and promising results were obtained.

D'Angelo et al. ${ }^{87}$ investigated the effects of CeONP protection of neuronal cells against cell death induced by Alzheimer's injury on an in vitro human $\mathrm{AD}$ model. $\mathrm{AD}$ is the most common neurodegenerative disorder and the primary cause of dementia in the elderly. It is characterized by cerebral extracellular amyloid plaques and intracellular neurofibrillary tangles formed by polymerization of amyloid $\beta$-peptides $(A \beta){ }^{88}$ It is associated with multiple etiologies and pathogenic mechanisms, and $A \beta$-associated free radicals and the resultant oxidative stress are an important part of the mechanism that is involved in the pathogenic cascade. ${ }^{89}$ Barbara et al. established the $\mathrm{AD}$ model by the $\mathrm{A} \beta$-treated differentiated SH-SY5Y human neuroblastoma cells. The A $\beta$-treated cells showed evident morphological alterations and axonal and dendritic dystrophy. However, incubation with CeONP protected cell viability and cell morphology from $\mathrm{A} \beta$ injury. Barbara et al. demonstrated that CeONP acted not only as a mere antioxidant agent but also as a modulator of signal transduction pathways for neuronal survival.

Subsequently, Cimini et al. ${ }^{90}$ developed an anti-amyloid $\beta$-antibody-conjugated CeONP to enhance its specific target to $A \beta$ aggregates. They synthesized polyethylene glycol-coated CeONP and subsequently conjugated anti-amyloid $\beta$-antibody to it (anti-A $\beta$ CEONP-polyethylene glycol). The results showed that the conjugation could be selectively delivered to the $A \beta$ plaques and there was a concomitant increase in neuronal survival (Figure 7A). This anti-A $\beta-C e O N P$ conjugation may be a potential candidate for antineurodegenerative therapy in vivo.

Kim et al. ${ }^{91}$ reported that CeONP can protect against ischemic stroke. Cerebral ischemia, or stroke, caused by a reduction of blood flow to the brain because of a clot or hemorrhage is the third leading cause of death worldwide. The lack of energy production that occurs as a result of the reduction in glucose and oxygen delivery to brain cells could lead to a disruption in ionic homeostasis and consequently induces excitotoxicity, oxidative stress, inflammation, BBB dysfunction and, ultimately, cell death. Kim et al. ${ }^{91}$ synthesized CeONP and encapsulated it with phospholipid-polyethylene glycol to enable longer circulation in the bloodstream by reducing nonspecific binding and uptake by organs. They demonstrated that CeONP could reduce ROS-induced cell death in vitro by using tertbutyl hydroperoxide treated $\mathrm{CHO}-\mathrm{K} 1$ cells. They further demonstrated this protective effect in living animal models (rats) with ischemic stroke. CeONP can reduce apoptotic cell death by decreasing ROS, which leads to a decreased infarct volume (Figure 7B).

Diabetes. Diabetes mellitus is a metabolic disorder characterized by hyperglycemia and insufficient secretion or action of endogenous insulin. ${ }^{92}$ Increased oxidative stress is a widely accepted participant in the development and progression of diabetes and its complications. ${ }^{92}$ Abdollahi and colleagues ${ }^{93}$ found that the combination of CeONP and sodium selenium was beneficial to diabetic rats. The weight of diabetic rats decreased significantly and blood glucose increased significantly compared with that in control rats. After treatment with the combination of sodium selenite and CeONP, a significant increase in weight and a significant decrease in blood glucose were shown. An improvement in biomarkers of diabetes, including oxidative stress, energy compensation (ADP/ATP), lipid profile and hepatic ROS levels, was also observed. This benefit effect was attributed to the synergistic antioxidant effect of CeONP and selenium.

Abdollahi and colleagues ${ }^{94}$ then reported that CeONP-sodium selenite combination could also improve pancreatic islet function during isolation and transplantation procedures. Patients with insulin-dependent diabetes mellitus require persistent insulin therapy, but pancreatic islet transplantation is usually the final cure that is needed in most cases. Despite recent improvements, islets of Langerhans experience excessive oxidative stress during isolation and transplantation procedures. Pretreatment with a CeONP-sodium selenite combination showed a significant increase in viability, secretion of insulin and ATP/ADP ratio, and a decrease in ROS levels in isolated, cultured pancreatic islets.

Retinal damage (retinal diseases). Photoreceptor cells have the highest rate of oxygen metabolism of any cells in the body and are continuously exposed to the deleterious effects of oxidative stress and constant bombardment by photons. The ROS that damage the sensitive cells in the retina are thought to have a central role in retinal diseases, including inherited retinal degeneration, diabetic retinopathy, macular degeneration and retinal detachment, which could lead to partial or complete loss of vision. ${ }^{95}$ McGinnis and coworkers ${ }^{96}$ demonstrated for the first time that CeONP could prevent retinal degeneration induced by intracellular peroxides and thus preserve retinal morphology and prevent loss of retinal function by using in vitro cell culture system and an in vivo albino rat lightdamage model (Figure 7C). Next, they explored the mechanism further by using the homozygous tubby mutant mouse as a model, which exhibits inherited early progressive cochlear and retinal degeneration. ${ }^{97,98}$ McGinnis and co-workers demonstrated that CeONP protects the retina by decreasing ROS, upregulating the expression of neuroprotection-associated genes, downregulating apoptosis signaling pathways and/or upregulating survival signaling pathways to slow photoreceptor degeneration. In a specific age-related macular degeneration model, Zhou et al. ${ }^{99}$ found that CeONP could inhibit rises in ROS, increases in vascular endothelial growth factor in the photoreceptor layer and the formation of intraretinal and subretinal neovascular lesions. CeONP could also induce the regression of pre-existing pathologic retinal neovasculature.

Chronic inflammation. Chronic inflammation is a complex immunological disorder that can result in irreversible organ damage, and often precedes and promotes the development of many major diseases. ${ }^{100}$ Overproduction of the free radical nitric oxide $(\cdot \mathrm{NO})$ has been implicated as a critical mediator of inflammation. The ability of CeONP to scavenge $\cdot \mathrm{NO}$ makes it a potential anti-inflammatory agent. Hirst et al. ${ }^{27}$ reported that CeONP could inhibit inflammatory mediator production in J774A.1 murine macrophages. In vivo studies also showed good biocompatibility of CeONP; it could be deposited in mouse tissues with no pathogenicity, and was well tolerated and incorporated into cellular tissues.

These studies suggest that CeONP has significant potential for therapeutic treatment of most types of oxidative-stress-related diseases. 
A
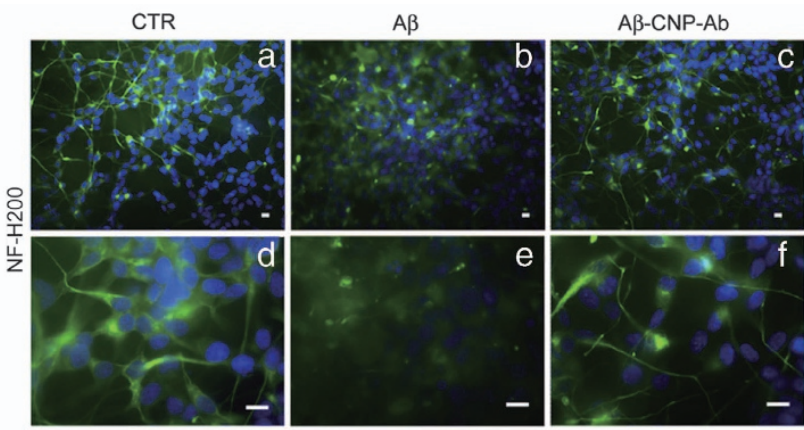

C

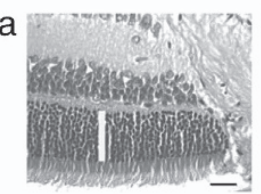

Control



$0.1 \mu \mathrm{M} \mathrm{CeO}{ }_{2}+\mathrm{LE}$

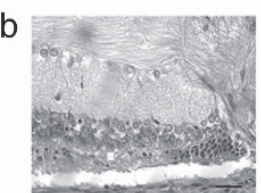

LE

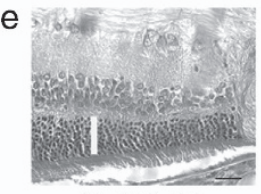

$0.3 \mu \mathrm{M} \mathrm{CeO}_{2}+\mathrm{LE}$

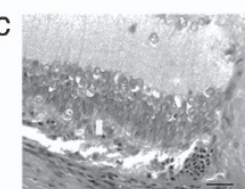

$0.9 \% \mathrm{NaCl}+\mathrm{LE}$

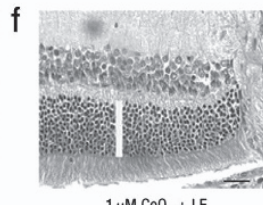

$1 \mu \mathrm{M} \mathrm{CeO}{ }_{2}+\mathrm{LE}$

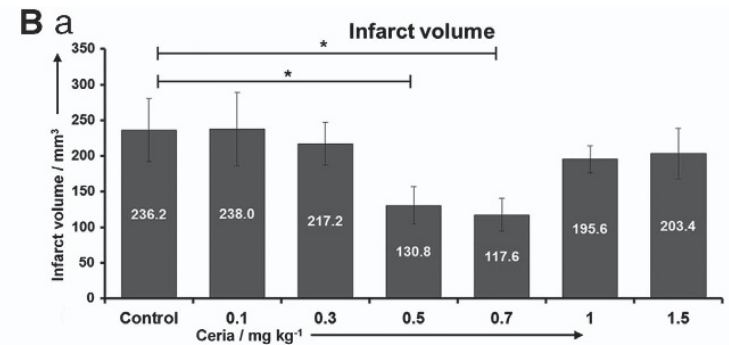

b

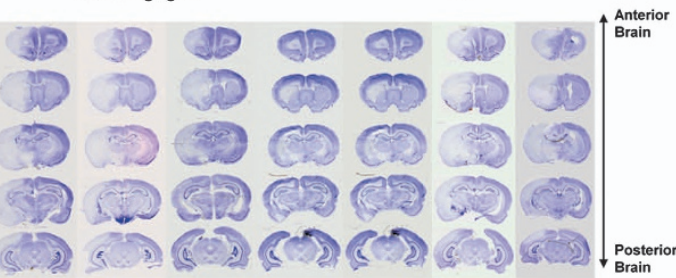

C

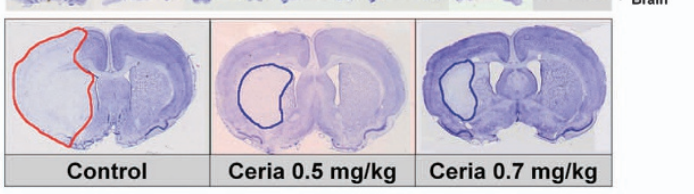

d

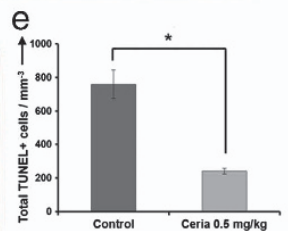

Figure 7 (A) The protective effect for in vitro Alzheimer's disease (AD) cell model (amyloid $\beta$-peptide (A $\beta$ )-treated human neuroblastoma-SH-SY5Y cells) by antibody-conjugated PEGylated cerium oxide nanoparticle (CeONP), which was evaluated by the immunofluorescence analysis for heavy neurofilament (NF-H 200 ) in control, $A \beta$-treated cells and $A \beta-C e O N P-A \beta$-treated cells. ${ }^{90}$ Copyright 2012 , Elsevier. (B) Comparison of brain infarct volumes in rats treated with various doses of CeONP. ${ }^{91}$ Copyright 2012, Wiley-VCH. (C) Intravitreal injection of CeONP protects rat retina photoreceptor cells from light-induced degeneration. LE, light exposure; Control, no LE, no injection. ${ }^{96}$ Copyright 2006, Nature Publishing Group.

Cancer. Previous studies have shown that CeONP is cytotoxic to cancer cells, inducing oxidative stress and causing lipid peroxidation and cell membrane leakage. ${ }^{101}$ It is also reported to protect normal cells but not cancer cells from ROS damage. ${ }^{29}$ This may be attributed to cancer cells having a more acidic cytosolic $\mathrm{pH}$ than normal cells because of higher glycolysis and significantly higher production of lactate. ${ }^{102}$ As previously mentioned, in acidic conditions, the antioxidant ability of CeONP is lost and it behaves as a strong oxidant, which may facilitate the oxidation of intracellular and extracellular components to induce cell apoptosis. CeONP was also reported to manipulate tumor-stromal interactions to detract tumor progression and invasion while benefiting stromal cells, ${ }^{103}$ which was attributed to the bifunctional character of CeONP. The cytosolic $\mathrm{pH}$ environments of stromal cells (for example, fibroblasts) are neutral; thus, CeONP would behave as an antioxidant to scavenge the ROS. Although epithelial/stromal signaling is largely mediated by myofibroblasts, and ROS have an important role in trigger mesenchymal-mesenchymal transition of human dermal fibroblasts to myofibroblasts, treatment with CeONP would downregulate the expression of myofibroblastic cells and inhibit the tumor invasion. Taken together, CeONP has both direct cytotoxicity and indirect antiinvasive properties on tumor cells.

Furthermore, CeONP is also shown to confer radioprotection to normal cells while increasing toxicity to cancer cells, ${ }^{104-107}$ which is significant in radiation therapy (RT). Although RT remains an effective modality for the control and cure of malignant cancer, many harmful side effects are associated with it, including fatigue, nausea and dermatitis. Many recent research studies have been focused on the alleviation of radiation damage on surrounding healthy cells. Amifostine $\mathrm{e}^{108}$ is the only clinically available radioprotectant, even though it can cause nausea and hypotension. The bifunctional character of CeONP gives it the potential to be used as an adjuvant for RT. Several publications have shown that treatment with CeONP before RT exposure decreases the RT-induced cell damage and death in normal tissues of the gastrointestinal tract, ${ }^{104}$ lung, ${ }^{105}$ breast, ${ }^{106}$ and head and neck. ${ }^{107}$ Conversely, CeONP could enhance the efficiency of RT to induce tumor cell death because of its acidic cytosolic environment. ${ }^{106,107}$

\section{Drug delivery devices and bioscaffold}

Recently, nanoparticles have shown tremendous potential use in biotechnology as drug delivery systems and bioscaffolds. CeONP, a nanomaterial with pharmacological potential, could be used as a nanocarrier or scaffold and also act as a therapeutic agent. Combining the two properties increases CeONP's potential application in biotechnology.

Drug delivery devices. Inspired by the distinctive and multifaceted properties of CeONP, a multifunctional CeONP-capped mesoporous silica nanoparticle (MSN) anticancer drug delivery system was designed by $\mathrm{Qu}$ and colleagues ${ }^{16}$ (Figure $8 \mathrm{~A}$ ). They synthesized $\beta$-cyclodextrin-modified CeONP and ferrocene-functionalized MSN. Under physiological conditions, $\beta$-cyclodextrin-modified CeONP could cap onto ferrocene-functionalized MSN through host-guest 
A

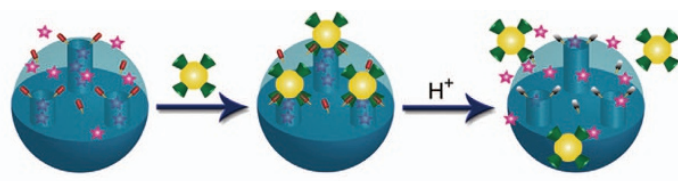



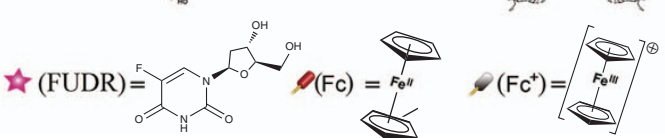

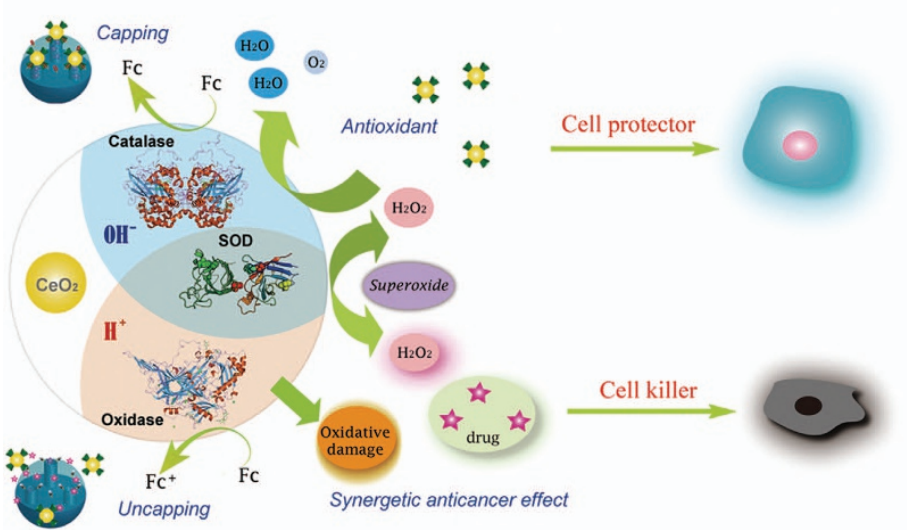

B
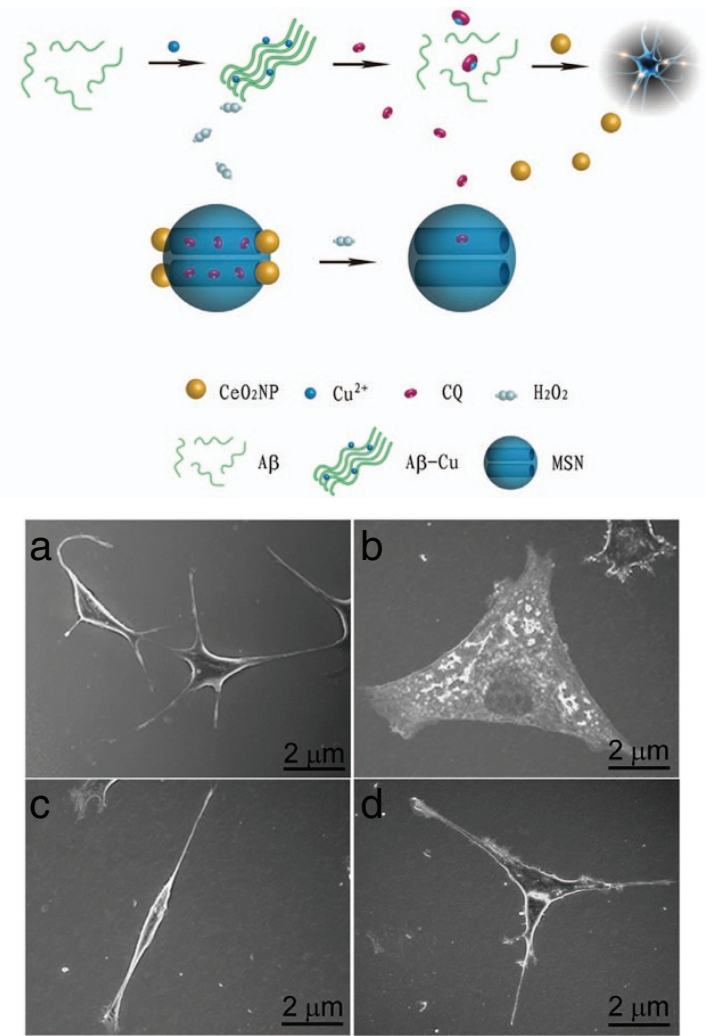

Figure 8 (A) (top) Schematic illustration for $\mathrm{pH}$-triggered release of the anticancer drug from $\beta$-cyclodextrin-modified cerium oxide nanoparticle ( $\beta$ CD@CeONP) capped ferrocene-functionalized mesoporous silica nanoparticle (Fc-MSN) to the cytosol. (below) The different enzymatic activities of CeONP at different $\mathrm{pH}$ values could make it be a cell protector or a cell killer. ${ }^{16}$ Copyright 2013 , Wiley- $\mathrm{VCH}$. (B) (top) Schematic representation of $\mathrm{H}_{2} \mathrm{O}_{2}$-fueled release of guest molecule clioquinol (CQ) from the pores of MSN capped with CeONP. CQ can sequester $\mathrm{Cu}^{2+}$ from amyloid $\beta$-peptide (A $\beta$ ), inhibiting aggregation. CeONP can protect cells from oxidative stress. Below: scanning electron microscopic images representing morphological observations performed on $\mathrm{A} \beta-\mathrm{Cu}^{2+}$-treated differentiated $\mathrm{PC} 12$ cells. (a) Control, (b) $\mathrm{A} \beta-\mathrm{Cu}^{2+}$ complex, (c) $\mathrm{A} \beta-\mathrm{Cu}^{2+}$ complex with glucose-coated CeONP (G-CeONP) and (d) $\mathrm{A} \beta-\mathrm{Cu}^{2+}$ complex with MSN-CQ-G-CeONP. ${ }^{17}$ Copyright 2013, Royal Society of Chemistry.

interactions between $\beta$-CD and ferrocene. After internalization into lung carcinoma (A549) cells by a lysosomal pathway $(\mathrm{pH} \approx 4.5-5.0$ ), the ferrocenyl moieties were oxidized to ferrocenium ions by CeONP lids, which triggered the uncapping of CeONP and caused the drug release. Because of the cytotoxicity of CeONP under acidic conditions, it exhibited a synergistic antitumor effect with the antitumor drugs. Furthermore, because of its antioxidant properties in physiological environments, CeONP would be beneficial in the next metabolic process. The multifunctional properties made CeONP an attractive drug delivery material.

By using the neuroprotection property and neurodegenerative disorder treatment ability of CeONP, Qu and colleagues ${ }^{17}$ reported a CeONP-capped, $\mathrm{H}_{2} \mathrm{O}_{2}$-responsive drug release system for $\mathrm{AD}$ treatment (Figure 8B). Orthodox metal chelators were developed to be used as therapeutic agents for $\mathrm{AD}$ to alleviate the metal-related toxicity. However, their applications were restricted by the poor BBB permeability and low inhibition efficiency. To this end, the authors intended to load metal chelators in a phenylboronic acid-functionalized MSN carrier sealed with glucose-coated CeONP through a unique covalent reaction of phenylboronic acid and glucose to form cyclic boronate moieties. ${ }^{17}$ The designed delivery platform could cross the BBB. The cyclic boronate moieties could be broken in the presence of $\mathrm{H}_{2} \mathrm{O}_{2}$ produced by $\mathrm{A} \beta$ aggregate metal ions, resulting in the release of CeONP and the guest molecules, which indicated that the platform could realize targeted drug delivery. Their design combined the anti-aggregation property of metal chelators and anti-oxidant property of CeONP in one system. This two-in-one bifunctional platform could effectively inhibit $A \beta$ aggregate formation, decrease cellular ROS and protect cells from $A \beta$-related toxicity (Figure $8 \mathrm{~B}$ ).

Bioscaffolds. Seal and co-workers ${ }^{18}$ reported that CeONP could increase the production of collagen by human mesenchymal stem cells (HMSCs) cultured on porous bioactive glass scaffolds. Porous bioactive glass scaffolds, which have been used clinically, can bind to bone and act as a temporary guide and stimulus for bone growth in three dimensions. Bone-marrow-derived HMSCs line is a crucial cell type for bone regeneration in vivo, because they differentiate to osteoprogenitor cells. It is reported that HMSCs are sensitive to toxic compounds derived from molecular oxygen. CeONP can increase the proliferation of HMSCs by neutralizing oxidative stress. Thus, embedding CeONP into porous three dimensional bioactive glass foam scaffolds could enhance osteoblastic differentiation of HMSCs and collagen formation.

By mixing CeONP powder and poly(D,L-lactic-co-glycolic acid) at specific concentrations followed by solvent casting onto prepatterned molds, Mandoli et al. ${ }^{19}$ fabricated hybrid two dimensional polymericceramic biosupports for stem cells culturing in vitro. CeONP not only improved the polymer-based composite mechanical properties but also stimulated the regenerating tissue biochemical activity, 

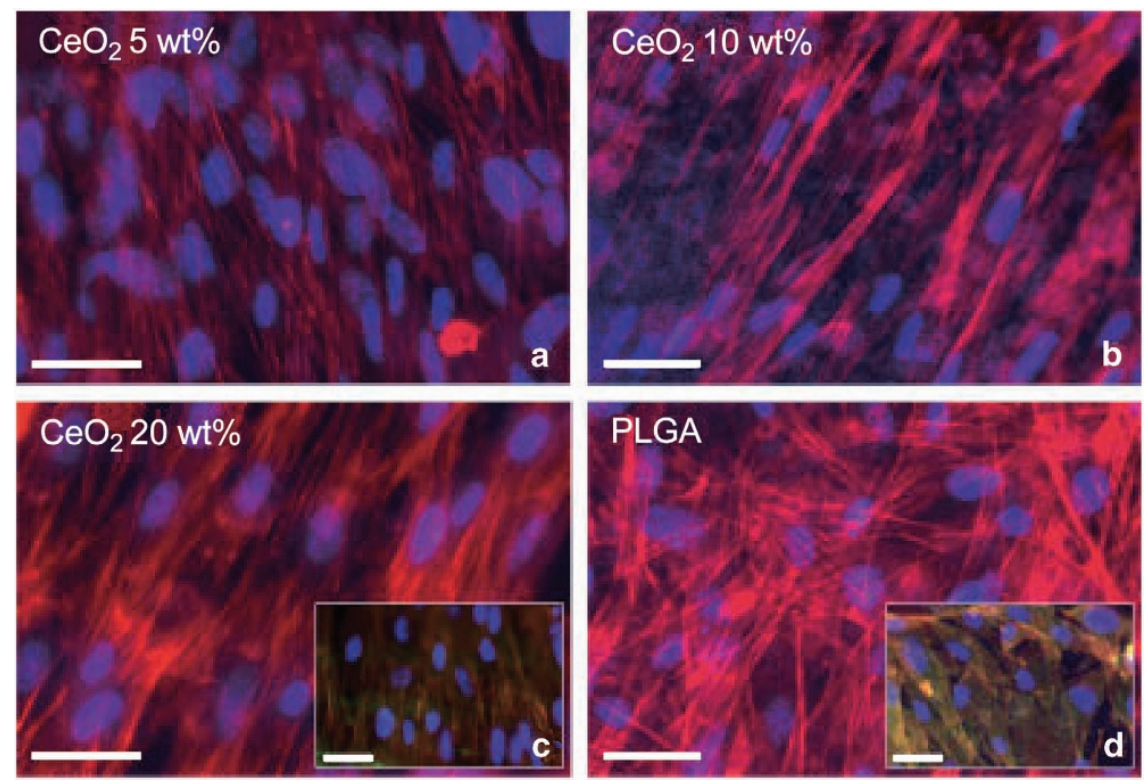

Figure 9 Immunofluorescence-culture monitoring of the murine resident adult cardiac stem cell seeded composite supports after 6 days in vitro: (a) 5 , (b) 10 and (c) 20 wt\% cerium oxide nanoparticle/poly(D,L-lactic-co-glycolic acid) (CeONP/PLGA), together with (d) unfilled PLGA as a control. ${ }^{19}$ Copyright 2010, Wiley-VCH.

contributing to its antioxidant activity. The effect of CeONP on the biological response might be ascribed either to the chemical nature of CeONP or from the physical and morphological changes in roughness and stiffness brought to the composite scaffolds (Figure 9). These findings showed that hybrid ceramic polymeric bioactive scaffolds loaded with CeONP hold great potential for tissue engineering applications.

\section{THE TOXICOLOGICAL EFFECTS OF CEONP}

Being a nanomaterial with numerous potential biological applications, the toxicological effects of CeONP have attracted more and more attention. A variety of in vitro and in vivo studies were carried out, and the results showed controversial conclusions.

\section{In vitro studies}

CeONP could be successful uptaken by mammalian cells in both normal and diseased states through multiple routes. ${ }^{77}$ In most cases of in vitro intracellular assays, CeONP was reported to exhibit positive effects (such as scavenging ROS) and was regarded as a promising biomaterial for biomedical applications. ${ }^{9}$ However, several reports suggested that the uptake of CeONP could induce oxidative stress and DNA damage, dephosphorylation of various substrates, aberrant cell signaling and alterations in the transcriptional and posttranslational levels. ${ }^{109-111}$ The cell lines used in the published studies established cell lines and primary cultures from tumors or normal tissues, which has been thoroughly reviewed in the literature. Although it was recently demonstrated that localization of CeONP in acidified lysosomes induced cell death ${ }^{111}$ and that CeONP could induce cytotoxicity through autophagy and a mitochondrial apoptosis pathway, ${ }^{110}$ simple correlations of the cytotoxicity effect of CeONP with cell types or particle size/preparations has not been established. ${ }^{112}$

\section{In vivo studies}

In vivo analyses of the biological effect of CeONP were carried out by peroral, intravenous or intraperitoneal administration to laboratory animals. It was reported that using CeONP as a food additive could increase the average and maximum lifespan of Drosophila. ${ }^{84}$ The potential pharmacological activity of CeONP has also been evaluated. It was shown that CeONP could prevent retinal degeneration induced by intracellular peroxides ${ }^{96}$ and could relieve the symptoms of ischemic stroke, ${ }^{91}$ ischemic cardiomyopathy, ${ }^{113}$ diabetes, ${ }^{93}$ radiation-induced pneumonitis ${ }^{105}$ and lupus ${ }^{84}$ in animal models of disease. These effects mainly depended on the ROS scavenging ability of CeONP. However, there were still a few studies showing that exposure of animals to CeONP resulted in significant lung responses, including lung inflammation, cytotoxicity, lung injury, alveolar macrophage functional changes, induction of phospholipidosis and release of pro-inflammatory and fibrotic cytokines. ${ }^{114}$ Cerium is also linked to fibrosis of the heart, and CeONP was shown to induce myocardial fibroblast proliferation and collagen deposition in rats. ${ }^{115}$ In addition, as cerium is not found in the human body and there are no clearance mechanisms for it, systemic toxicity would be the result.

\section{CONCLUSIONS AND PERSPECTIVES}

Derived from quick and expedient mutations of the oxidation state between $\mathrm{Ce}^{4+}$ and $\mathrm{Ce}^{3+}$, CeONP has excellent catalytic and multienzyme-mimetic properties. These make it attractive for widespread applications, both in industry and in biosystems. At present, the industrial applications of CeONP are well developed, whereas bioapplications are still in their infancy. Numerous studies have reported enzyme-like activities of CeONP, the results of which were supported by abiotic studies in simple buffer solutions; however, they must be demonstrated and investigated further in biological media, cells, tissues and even animals. In addition, there are divergent biological effects obtained with CeONP, with it being beneficial in one case and toxic in another. Thus, the toxic mechanism should be carefully and systematically investigated with animal models over long periods of time, and comprehensive investigative methods must be developed. It is also worth noting that the CeONP used in the investigations were 
not consist in preparation, particle size or surface characteristic, even though these features may have important role in CeONP's biological reactivity/toxicology. Unfortunately, until recently, information about the relationships between the properties of CeONP was fragmented and vague. Further systematic investigations are required.

Although there are still some unresolved issues and challenges, the unique physical and chemical properties of CeONP and the achieved significant advances of it clearly demonstrate that CeONP is a fascinating and versatile material that is promising for numerous industrial and biomedical applications.

\section{ACKNOWLEDGEMENTS}

This work was supported by 973 Project (2011CB936004) and NSFC (21210002, 91213302).

1 Hu, Z., Haneklaus, S., Sparovek, G. \& Schnug, E. Rare earth elements in soils. Commun. Soil Sci. Plant. Anal. 37, 1381-1420 (2006).

2 Bouzigues, C., Gacoin, T. \& Alexandrou, A. Biological applications of rare-earth based nanoparticles. Acs Nano 5, 8488-8505 (2011).

3 Conesa, J. C. computer modeling of surfaces and defects on cerium dioxide. Surf. Sci. 339, 337-352 (1995)

4 Stambouli, A. B. \& Traversa, E. Solid oxide fuel cells (SOFCs): a review of an environmentally clean and efficient source of energy. Renew. Sust. Energ. Rev. 6 , 433-455 (2002).

5 Patil, S., Kuiry, S. C., Seal, S. \& Vanfleet, R. Synthesis of nanocrystalline ceria particles for high temperature oxidation resistant coating. J. Nanopart. Res. 4 433-438 (2002).

6 Trovarelli, A. Catalytic properties of ceria and $\mathrm{CeO}_{2}$-containing materials. Catal. Rev. 38, 439-520 (1996).

7 Kaspar, J., Fornasiero, P. \& Graziani, M. Use of $\mathrm{CeO}_{2}$-based oxides in the three-way catalysis. Catal. Today 50, 285-298 (1999).

8 Corma, A., Atienzar, P., Garcia, H. \& Chane-Ching, J. Y. Hierarchically mesostructured doped $\mathrm{CeO}_{2}$ with potential for solar-cell use. Nat. Mater. 3, 394-397 (2004).

9 Celardo, I., Pedersen, J. Z., Traversa, E. \& Ghibelli, L. Pharmacological potential of cerium oxide nanoparticles. Nanoscale 3, 1411-1420 (2011)

10 Asati, A., Santra, S., Kaittanis, C., Nath, S. \& Perez, J. M. Oxidase-like activity of polymer-coated cerium oxide nanoparticles. Angew. Chem. Int. Ed. 48, 2308-2312 (2009)

11 Asati, A., Kaittanis, C., Santra, S. \& Perez, J. M. pH-tunable oxidase-like activity of cerium oxide nanoparticles achieving sensitive fluorigenic detection of cance biomarkers at neutral pH. Anal. Chem. 83, 2547-2553 (2011).

$12 \mathrm{Li}$, X., Sun, L., Ge, A. \& Guo, Y. Enhanced chemiluminescence detection of thrombin based on cerium oxide nanoparticles. Chem. Commun. 47, 947-949 (2011).

13 Kaittanis, C., Santra, S., Asati, A. \& Perez, J. M. A cerium oxide nanoparticle-based device for the detection of chronic inflammation via optical and magnetic resonance imaging. Nanoscale 2117-2123 (2012).

14 Ornatska, M., Sharpe, E., Andreescu, D. \& Andreescu, S. Paper bioassay based on ceria nanoparticles as colorimetric probes. Anal. Chem. 83, 4273-4280 (2011).

15 Lin, Y. H., Xu, C., Ren, J. S. \& Qu, X. G. Using thermally regenerable cerium oxide nanoparticles in biocomputing to perform label-free, resettable, and colorimetric logic operations. Angew. Chem. Int. Ed. 51, 12579-12583 (2012).

16 Xu, C., Lin, Y., Wang, J., Wu, L., Wei, W., Ren, J. \& Qu, X. Nanoceria-triggered synergetic drug release based on $\mathrm{CeO}_{2}$-capped mesoporous silica host-guest interactions and switchable enzymatic activity and cellular effects of $\mathrm{CeO}_{2}$. Adv. Healthcare Mater. 2, 1591-1599 (2013).

17 Li, M., Shi, P., Xu, C., Ren, J. S. \& Qu, X. G. Cerium oxide caged metal chelator anti-aggregation and anti-oxidation integrated $\mathrm{H}_{2} \mathrm{O}_{2}$-responsive controlled drug release for potential Alzheimer's disease treatment. Chem. Sci. 4, 2536-2542 (2013).

18 Karakoti, A. S., Tsigkou, O., Yue, S., Lee, P. D., Stevens, M. M., Jones, J. R. \& Seal, S. Rare earth oxides as nanoadditives in 3-D nanocomposite scaffolds for bone regeneration. J. Mater. Chem. 20, 8912-8919 (2010).

19 Mandoli, C., Pagliari, F., Pagliari, S., Forte, G., Di Nardo, P., Licoccia, S. \& Traversa, E. Stem cell aligned growth induced by $\mathrm{CeO}_{2}$ nanoparticles in PLGA scaffolds with improved bioactivity for regenerative medicine. Adv. Funct. Mater. 20, 1617-1624 (2010)

20 Yu, S. H., Colfen, H. \& Fischer, A. High quality $\mathrm{CeO}_{2}$ nanocrystals stabilized by a double hydrophilic block copolymer. Colloid Surf. A 234, 49-52 (2004).

21 Inoue, M., Kimura, M. \& Inui, T. Transparent colloidal solution of $2 \mathrm{~nm}$ ceria particles. Chem. Commun. 957-958 (1999).

$22 \mathrm{Gu}, \mathrm{H}$. \& Soucek, M. D. Preparation and characterization of monodispers cerium oxide nanoparticles in hydrocarbon solvents. Chem. Mater. 19, 1103-1110 (2007)
23 Sreeremya, T. S., Thulasi, K. M., Krishnan, A. \& Ghosh, S. A novel aqueous route to fabricate ultrasmall monodisperse lipophilic cerium oxide nanoparticles. Ind. Eng. Chem. Res. 51, 318-326 (2012).

24 Sathyamurthy, S., Leonard, K. J., Dabestani, R. T. \& Paranthaman, M. P. Reverse micellar synthesis of cerium oxide nanoparticles. Nanotechnology 16, 1960-1964 (2005).

25 Lin, H. L., Wu, C. Y. \& Chiang, R. K. Facile synthesis of $\mathrm{CeO}_{2}$ nanoplates and nanorods by [100] oriented growth. J. Colloid Interf. Sci. 341, 12-17 (2010).

26 Madler, L., Stark, W. J. \& Pratsinis, S. E. Flame-made ceria nanoparticles. J. Mater Res. 17, 1356-1362 (2002).

27 Hirst, S. M., Karakoti, A. S., Tyler, R. D., Sriranganathan, N., Seal, S. \& Reilly, C. M Anti-inflammatory properties of cerium oxide nanoparticles. Small 24, 2848-2856 (2009).

28 Karakoti, A. S., Singh, S., Kumar, A., Malinska, M., Kuchibhatla, S. V. N. T. Wozniak, K., Self, W. T. \& Seal, S. PEGylated nanoceria as radical scavenger with tunable redox chemistry. J. Am. Chem. Soc. 131, 14144-14145 (2009).

29 Perez, J. M., Asati, A., Nath, S. \& Kaittanis, C. Synthesis of biocompatible dextrancoated nanoceria with $\mathrm{pH}$-dependent antioxidant properties. Small 4, 552-556 (2008).

30 Suzuki, T., Kosacki, I., Anderson, H. U. \& Colomban, P. Electrical conductivity and lattice defects in nanocrystalline cerium oxide thin films. J. Am. Ceram. Soc. 84, 2007-2014 (2001).

31 Zhang, F., Chen, C.-H., Raitano, J. M., Hanson, J. C., Caliebe, W. A., Khalid, S. \& Chan, S.-W. Phase stability in ceria-zirconia binary oxide nanoparticles: the effect of the $\mathrm{Ce}^{3+}$ concentration and the redox environment. J. Appl. Phys. 99, 084313 (2006).

32 Dutta, P., Pal, S., Seehra, M. S., Shi, Y., Eyring, E. M. \& Ernst, R. D. Concentration of $\mathrm{Ce}^{3+}$ and oxygen vacancies in cerium oxide nanoparticles. Chem. Mater. 18, 5144-5146 (2006).

33 Deshpande, S., Patil, S., Kuchibhatla, S. V. N. T. \& Seal, S. Size dependency variation in lattice parameter and valency states in nanocrystalline cerium oxide. Appl. Phys. Lett. 87, 133113 (2005).

34 Kuchma, M. H., Komanski, C. B., Colon, J., Teblum, A., Masunov, A. E., Alvarado, B. Babu, S., Seal, S., Summy, J. \& Baker, C. H. Phosphate ester hydrolysis of biologically relevant molecules by cerium oxide nanoparticles. Nanomed. Nanotechnol. 6, 738-744 (2010)

35 Skorodumova, N. V. Simak, S. I. Lundqvist, B I. Abrikosov, I. A \& Johansson, B. Quantum origin of the oxygen storage capability of ceria. Phys. Rev. Lett. 89, 166601 (2002).

36 Romeo, M., Bak, K., Elfallah, J., Lenormand, F. \& Hilaire, L. Xps study of the reduction of cerium dioxide. Surf. Interface Anal. 20, 508-512 (1993).

37 Binet, C., Badri, A. \& Lavalley, J. C. A spectroscopic characterization of the reduction of ceria from electronic-transitions of intrinsic point-defects. J. Phys. Chem. 98, 6392-6398 (1994).

38 Binet, C., Daturi, M. \& Lavalley, J. C. IR study of polycrystalline ceria properties in oxidised and reduced states. Catal. Today 50, 207-225 (1999).

39 Matsumoto, M. Soda, K., Ichikawa, K., Tanaka, S., Taguchi, Y, Jouda, K., Aita, 0. Tezuka, Y. \& Shin, S. Resonant photoemission-study of $\mathrm{CeO}_{2}$. Phys. Rev. B 50, 11340-11346 (1994).

40 Fukui, K., Namai, Y. \& Iwasawa, Y. Imaging of surface oxygen atoms and their defect structures on $\mathrm{CeO}_{2}(111)$ by noncontact atomic force microscopy. Appl. Surf. Sci. 188, 252-256 (2002).

41 Namai, Y., Fukui, K. \& Iwasawa, Y. Atom-resolved noncontact atomic force microscopic observations of $\mathrm{CeO}_{2}(111)$ surfaces with different oxidation states: Surface structure and behavior of surface oxygen atoms. J. Phys. Chem. B 107, 11666-11673 (2003)

42 Esch, F., Fabris, S., Zhou, L., Montini, T., Africh, C., Fornasiero, P., Comelli, G. \& Rosei, R. Electron localization determines defect formation on ceria substrates. Science 309, 752-755 (2005).

43 Korsvik, C., Patil, S., Seal, S. \& Self, W. T. Superoxide dismutase mimetic properties exhibited by vacancy engineered ceria nanoparticles. Chem. Commun. 1056-1058 (2007).

44 Pirmohamed, T., Dowding, J. M., Singh, S., Wasserman, B., Heckert, E., Karakoti, A S., King, J. E. S., Seal, S. \& Self, W. T. Nanoceria exhibit redox state-dependent catalase mimetic activity. Chem. Commun. 46, 2736-2738 (2010).

45 Jiao, X., Song, H. J., Zhao, H. H., Bai, W., Zhang, L. C. \& Lv, Y. Well-redispersed ceria nanoparticles: promising peroxidase mimetics for $\mathrm{H}_{2} \mathrm{O}_{2}$ and glucose detection. Anal. Methods 4, 3261-3267 (2012).

46 Buettner, G. R. Superoxide dismutase in redox biology: the roles of superoxide and hydrogen peroxide. Anticancer Agent Med. Chem. 11, 341-346 (2011)

47 Heckert, E. G., Karakoti, A. S. Seal, S. \& Self, W. T. The role of cerium redox state in the SOD mimetic activity of nanoceria. Biomaterials 29, 2705-2709 (2008)

48 Nicholls, P. Classical catalase: Ancient and modern. Arch. Biochem. Biophys. 525, 95-101 (2012).

49 Lipinski, B. Hydroxyl radical and its scavengers in health and disease. Oxid. Med. Cell Longev. 2011, 809696 (2011)

50 Xue, Y., Luan, Q. F., Yang, D., Yao, X. \& Zhou, K. B. Direct evidence for hydroxy radical scavenging activity of cerium oxide nanoparticles. J. Phys. Chem. C 115, 4433-4438 (2011).

51 Singh, S., Dosani, T., Karakoti, A. S., Kumar, A., Seal, S. \& Self, W. T. A phosphatedependent shift in redox state of cerium oxide nanoparticles and its effects on catalytic properties. Biomaterials 32, 6745-6753 (2011). 
52 Alili, L., Sack, M., Karakoti, A. S., Teuber, S., Puschmann, K., Hirst, S. M., Reilly, C. M., Zanger, K., Stahl, W., Das, S., Seal, S. \& Brenneisen, P. Combined cytotoxic and anti-invasive properties of redox-active nanoparticles in tumor-stroma interactions. Biomaterials 32, 2918-2929 (2011).

53 Das, M., Patil, S., Bhargava, N., Kang, J. F., Riedel, L. M., Seal, S. \& Hickman, J. J. Auto-catalytic ceria nanoparticles offer neuroprotection to adult rat spinal cord neurons. Biomaterials 28, 1918-1925 (2007)

54 Knott, A. B. \& Bossy-Wetzel, E. Nitric oxide in health and disease of the nervous system. Antioxid. Redox Sign. 11, 541-553 (2009).

55 Martinezarias, A., Soria, J., Conesa, J. C., Seoane, X. L., Arcoya, A. \& Cataluna, R. no reaction at surface oxygen vacancies generated in cerium oxide. J. Chem. Soc., Faraday Trans. (1995)91, 1679-1687.

56 Dowding, J. M., Dosani, T., Kumar, A., Seal, S. \& Self, W. T. Cerium oxide nanoparticles scavenge nitric oxide radical ((NO)-N-center dot. Chem. Commun. 48, 4896-4898 (2012)

57 Wayland, B. B. \& Olson, L. W. Spectroscopic studies and bonding model for nitric oxide complexes of iron porphyrins. J. Am. Chem. Soc. 96, 6037-6041 (1974).

58 Azevedo, A. M., Martins, V. C., Prazeres, D. M. F., Vojinović, V., Cabral, J. M. S. \& Fonseca, L. P. Horseradish peroxidase: a valuable tool in biotechnology. In Biotechnology Annual Review (ed. El-Gewely, R.) Ch. 9, 299-332 (Elsevier, New York, 2003).

59 Gao, L. Z., Zhuang, J., Nie, L., Zhang, J. B., Zhang, Y., Gu, N., Wang, T. H., Feng, J., Yang, D. L., Perrett, S. \& Yan, X. Intrinsic peroxidase-like activity of ferromagnetic nanoparticles. Nat. Nanotechnol. 2, 577-583 (2007).

60 Song, Y. J., Qu, K. G., Zhao, C., Ren, J. S. \& Qu, X. G. Graphene oxide: intrinsic peroxidase catalytic activity and its application to glucose detection. Adv. Mater. 22, 2206-2210 (2010)

61 Heckert, E. G., Seal, S. \& Self, W. T. Fenton-like reaction catalyzed by the rare earth inner transition metal cerium. Environ. Sci. Technol. 42, 5014-5019 (2008).

62 Lee, H. J., Reimann, J., Huang, Y. F. \& Adelroth, P. Functional proton transfer pathways in the heme-copper oxidase superfamily. Biochim. Biophys. Acta 1817, 537-544 (2012)

63 Peng, Y. F., Chen, X. J., Yi, G. S. \& Gao, Z. Q. Mechanism of the oxidation of organic dyes in the presence of nanoceria. Chem. Commun. 47, 2916-2918 (2011).

64 Di Monte, R. \& Kaspar, J. On the role of oxygen storage in three-way catalysis. Top. Catal. 28, 47-57 (2004).

65 Cohen, P. The origins of protein phosphorylation. Nat. Cell Biol. 4, E127-E130 (2002).

66 Chin, J. Artificial dinuclear phosphoesterases. Curr. Opin. Chem. Biol. 1, 514-521 (1997).

67 Franklin, S. J. Lanthanide-mediated DNA hydrolysis. Curr. Opin. Chem. Biol. 5, 201-208 (2001)

68 Tan, F., Zhang, Y. J., Wang, J. L., Wei, J. Y., Cai, Y. \& Qian, X. H. An efficient method for dephosphorylation of phosphopeptides by cerium oxide. J. Mass Spectrom. 43, 628-632 (2008)

69 Patil, A J., Kumar, R. K., Barron, N. J. \& Mann, S. Cerium oxide nanoparticlemediated self-assembly of hybrid supramolecular hydrogels. Chem. Commun. 48, 7934-7936 (2012)

70 Penn, S. G., He, L. \& Natan, M. J. Nanoparticles for bioanalysis. Curr. Opin. Chem. Biol. 7, 609-615 (2003).

71 Xu, C., Liu, Z., Wu, L., Ren, J. \& Qu, X. Nucleoside triphosphates as promoters to enhance nanoceria enzyme-like activity and for single-nucleotide polymorphism typing. Adv. Funct. Mater. (2013) doi:10.1002/adfm.201301649.

72 Guliyev, R., Ozturk, S., Kostereli, Z. \& Akkaya, E. U. From virtual to physical: integration of chemical logic gates. Angew. Chem. Int. Ed. 50, 9826-9831 (2011).

73 Jakupec, M. A., Unfried, P. \& Keppler, B. K. Pharmacological properties of cerium compounds. Rev. Physiol. Bioch. P 153, 101-111 (2005).

74 Gordh, T. \& Rydin, H. the question of cerium oxalate as a prophylactic against postoperative vomiting. Anesthesiology 7, 526-535 (1946).

75 Courtiss, E. H. \& Monafo, W. W. Cerium nitrate: a new topical antiseptic for extensive burns. Plast. Reconstr. Surg. 59, 776 (1977).

76 Biba, F., Groessl, M., Egger, A., Jakupec, M. A. \& Keppler, B. K. A novel cytotoxic cerium complex: aquatrichloridobis(1,10-phenanthroline)cerium(III) (KP776). synthesis, characterization, behavior in $\mathrm{H}_{2} \mathrm{O}$, binding towards biomolecules, and antiproliferative activity. Chem. Biodivers. 6, 2153-2165 (2009).

77 Wason, M. S. \& Zhao, J. H. Cerium oxide nanoparticles: potential applications for cancer and other diseases. Am. J. Transl. Res. 5, 126-131 (2013).

78 Apel, K. \& Hirt, H. Reactive oxygen species: metabolism, oxidative stress, and signal transduction. Annu. Rev. Plant. Biol. 55, 373-399 (2004).

79 Mariani, E., Polidori, M. C., Cherubini, A. \& Mecocci, P. Oxidative stress in brain aging, neurodegenerative and vascular diseases: An overview. J. Chromatogr. B 827, 65-75 (2005)

80 Emerit, J., Edeas, M. \& Bricaire, F. Neurodegenerative diseases and oxidative stress. Biomed. Pharmacother. 58, 39-46 (2004).

81 Pardridge, W. M. Alzheimer's disease drug development and the problem of the bloodbrain barrier. Alzheimers Dement. 5, 427-432 (2009)

82 Karakoti, A., Singh, S., Dowding, J. M., Seal, S. \& Self, W. T. Redox-active radical scavenging nanomaterials. Chem. Soc. Rev. 39, 4422-4432 (2010).

83 Rzigalinski, B. A. Nanoparticles and cell longevity. Technol. Cancer Res. Treat. 4, 651-659 (2005).

84 Rzigalinski, B. A., Meehan, K., Davis, R. M., Xu, Y., Miles, W. C. \& Cohen, C. A. Radical nanomedicine. Nanomedicine 1, 399-412 (2006).
85 Schubert, D., Dargusch, R., Raitano, J. \& Chan, S. W. Cerium and yttrium oxide nanoparticles are neuroprotective. Biochem. Biophys. Res. Commun. 342, 86-91 (2006)

86 Hickman, J. J., Das, M., Patil, S., Bhargava, N., Kang, J. F., Riedel, L. \& Seal, S. Auto-catalytic Ceria nanoparticles offer neuroprotection to adult rat spinal cord neurons. Tissue Eng. 13, 873-874 (2007).

87 D’Angelo, B., Santucci, S., Benedetti, E., Di Loreto, S., Phani, R. A., Falone, S., Amicarelli, F., Ceru, M. P. \& Cimini, A. Cerium oxide nanoparticles trigger neuronal survival in a human alzheimer disease model by modulating BDNF pathway. Curr. Nanosci. 5, 167-176 (2009).

88 Geng, J., Li, M., Ren, J., Wang, E. \& Qu, X. Polyoxometalates as inhibitors of the aggregation of amyloid $\beta$ peptides associated with Alzheimer's disease. Angew. Chem. Int. Ed. 50, 4184-4188 (2011).

89 Varadarajan, S., Yatin, S., Aksenova, M. \& Butterfield, D. A. Review: Alzheimer's amyloid $\beta$-peptide-associated free radical oxidative stress and neurotoxicity. J. Struct. Biol. 130, 184-208 (2000).

90 Cimini, A., D’Angelo, B., Das, S., Gentile, R., Benedetti, E., Singh, V., Monaco, A. M., Santucci, S. \& Seal, S. Antibody-conjugated PEGylated cerium oxide nanoparticles for specific targeting of $A \beta$ aggregates modulate neuronal survival pathways. Acta Biomater. 8, 2056-2067 (2012)

91 Kim, C. K., Kim, T., Choi, I. Y., Soh, M., Kim, D., Kim, Y. J., Jang, H., Yang, H. S., Kim, J. Y., Park, H. K., Park, S. P., Park, S., Yu, T., Yoon, B. W., Lee, S. H. \& Hyeon, T. Ceria nanoparticles that can protect against ischemic stroke. Angew. Chem. Int. Ed. 51, 11039-11043 (2012).

92 Maritim, A. C., Sanders, R. A. \& Watkins, J. B. Diabetes, oxidative stress, and antioxidants: a review. J. Biochem. Mol. Toxicol. 17, 24-38 (2003).

93 Pourkhalili, N., Hosseini, A., Nili-Ahmadabadi, A., Hassani, S., Pakzad, M., Baeeri, M., Mohammadirad, A. \& Abdollahi, M. Biochemical and cellular evidence of the benefit of a combination of cerium oxide nanoparticles and selenium to diabetic rats. World J. Diabetes 2, 204-210 (2011).

94 Pourkhalili, N., Hosseini, A., Nili-Ahmadabadi, A., Rahimifard, M., Navaei-Nigjeh, M., Hassani, S., Baeeri, M. \& Abdollahi, M. Improvement of isolated rat pancreatic islets function by combination of cerium oxide nanoparticles/sodium selenite through reduction of oxidative stress. Toxicol. Mech. Methods 22, 476-482 (2012)

95 Sanvicens, N., Gomez-Vicente, V., Masip, I., Messeguer, A. \& Cotter, T. G. Oxidative stress-induced apoptosis in retinal photoreceptor cells is mediated by calpains and caspases and blocked by the oxygen radical scavenger CR-6. J. Biol. Chem. 279, 39268-39278 (2004).

96 Chen, J. P., Patil, S., Seal, S. \& McGinnis, J. F. Rare earth nanoparticles prevent retinal degeneration induced by intracellular peroxides. Nat. Nanotechnol. 1, 142-150 (2006)

97 Kong, L., Cai, X., Zhou, X. H., Wong, L. L., Karakoti, A. S., Seal, S. \& McGinnis, J. F. Nanoceria extend photoreceptor cell lifespan in tubby mice by modulation of apoptosis/survival signaling pathways. Neurobiol. Dis. 42, 514-523 (2011)

98 Cai, X., Sezate, S. A., Seal, S. \& McGinnis, J. F. Sustained protection against photoreceptor degeneration in tubby mice by intravitreal injection of nanoceria. Biomaterials 33, 8771-8781 (2012)

99 Zhou, X. H., Wong, L. L., Karakoti, A. S., Seal, S. \& McGinnis, J. F. Nanoceria inhibit the development and promote the regression of pathologic retinal neovascularization in the vldlr knockout mouse. PLoS One 6, e16733 (2011).

100 Federico, A., Morgillo, F., Tuccillo, C., Ciardiello, F. \& Loguercio, C. Chronic inflammation and oxidative stress in human carcinogenesis. Int. J. Cancer 121, 2381-2386 (2007).

101 Lin, W. S., Huang, Y. W., Zhou, X. D. \& Ma, Y. F. Toxicity of cerium oxide nanoparticles in human lung cancer cells. Int. J. Toxicol. 25, 451-457 (2006).

102 Ristow, M. Oxidative metabolism in cancer growth. Curr. Opin. Clin. Nutr. Metab. Care 9, 339-345 (2006).

103 Alili, L., Sack, M., Karakoti, A. S., Teuber, S., Puschmann, K., Hirst, S. M., Reilly, C. M., Zanger, K., Stahl, W., Das, S., Seal, S. \& Brenneisen, P. Combined cytotoxic and anti-invasive properties of redox-active nanoparticles in tumor-stroma interactions. Biomaterials 32, 2918-2929 (2011).

104 Colon, J., Hsieh, N., Ferguson, A., Kupelian, P., Seal, S., Jenkins, D. W. \& Baker, C. $\mathrm{H}$. Cerium oxide nanoparticles protect gastrointestinal epithelium from radiationinduced damage by reduction of reactive oxygen species and upregulation of superoxide dismutase 2. Nanomedicine 6, 698-705 (2010).

105 Colon, J., Herrera, L., Smith, J., Patil, S., Komanski, C., Kupelian, P., Seal, S., Jenkins, D. W. \& Baker, C. H. Protection from radiation-induced pneumonitis using cerium oxide nanoparticles. Nanomedicine 5, 225-231 (2009).

106 Tarnuzzer, R. W., Colon, J., Patil, S. \& Seal, S. Vacancy engineered ceria nanostructures for protection from radiation-induced cellular damage. Nano Lett. 5, 2573-2577 (2005).

107 Madero-Visbal, R. A., Alvarado, B. E., Colon, J. F., Baker, C. H., Wason, M. S., Isley, B., Seal, S., Lee, C. M., Das, S. \& Manon, R. Harnessing nanoparticles to improve toxicity after head and neck radiation. Nanomedicine 8, 1223-1231 (2012).

108 Citrin, D., Cotrim, A. P., Hyodo, F., Baum, B. J., Krishna, M. C. \& Mitchell, J. B. Radioprotectors and mitigators of radiation-induced normal tissue injury. Oncologist 15, 360-371 (2010).

109 Park, E. J., Choi, J., Park, Y. K. \& Park, K. Oxidative stress induced by cerium oxide nanoparticles in cultured BEAS-2B cells. Toxicology 245, 90-100 (2008).

110 Hussain, S., Al-Nsour, F., Rice, A. B., Marshburn, J., Yingling, B., Ji, Z. X., Zink, J. I., Walker, N. J. \& Garantziotis, S. Cerium dioxide nanoparticles induce apoptosis and autophagy in human peripheral blood monocytes. ACS Nano 6, 5820-5829 (2012). 
111 Cheng, G. L., Guo, W., Han, L., Chen, E. L., Kong, L. F., Wang, L. L., Ai, W. C., Song, N. N., Li, H. S. \& Chen, H. M. Cerium oxide nanoparticles induce cytotoxicity in human hepatoma SMMC-7721 cells via oxidative stress and the activation of MAPK signaling pathways. Toxicol. In Vitro 27, 1082-1088 (2013).

112 Asati, A., Santra, S., Kaittanis, C. \& Perez, J. M. Surface-charge-dependent cell localization and cytotoxicity of cerium oxide nanoparticles. ACS Nano 4, 5321-5331 (2010).

113 Niu, J. L., Azfer, A., Rogers, L. M., Wang, X. H. \& Kolattukudy, P. E. Cardioprotective effects of cerium oxide nanoparticles in a transgenic murine model of cardiomyopathy. Cardiovasc. Res. 73, 549-559 (2007).

114 Ma, J. Y., Zhao, H. W., Mercer, R. R., Barger, M., Rao, M., Meighan, T., SchweglerBerry, D., Castranova, V. \& Ma, J. K. Cerium oxide nanoparticle-induced pulmonary inflammation and alveolar macrophage functional change in rats. Nanotoxicology 5 , 312-325 (2011).

115 Ma, J. Y., Mercer, R. R., Barger, M., Schwegler-Berry, D., Scabilloni, J., Ma, J. K. \& Castranova, V. Induction of pulmonary fibrosis by cerium oxide nanoparticles. Toxicol. Appl. Pharm. 262, 255-264 (2012).

(c) (i) $(9)$ This work is licensed under a Creative Commons Attribution-NonCommercial-NoDerivs 3.0 Unported License. To view a copy of this license, visit http://creativecommons. org/licenses/by-nc-nd/3.0/

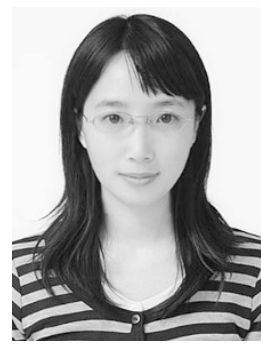

Can Xu received her BS from China University of Mining and Technology in 2008. She is now a PhD candidate majoring in Chemical Biology under the supervision of Professor Xiaogang Qu at the Changchun Institute of Applied Chemistry, Chinese Academy of Sciences (CAS). Her PhD is focused on expanding the horizons of biosensors and drug delivery systems based on artificial nano enzymes.

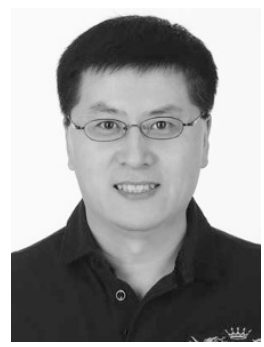

Xiaogang Qu received his PhD from the Chinese Academy of Sciences (CAS) in 1995 with CAS President's Award. He moved to the United States afterwards and worked with Professor JB Chaires in Mississippi Medical Center and Nobel Laureate Professor Ahmed H Zewail at the California Institute of Technology. Since late 2002, he is a professor at the Changchun Institute of Applied Chemistry, CAS. From December 2006 to May 2007, he visited Nobel Laureate Professor Alan J Heeger group in UCSB. His current research is focused on ligand-nucleic acids or related protein interactions, and biofunctional materials for advanced medical technology. 